\title{
Projective Multiresolution Analyses over Irrational Rotation Algebras
}

by

\section{Benjamin A. Purkis}

B.A., Amherst College, 2007

M.A., University of Colorado, Boulder, 2010

\author{
A thesis submitted to the \\ Faculty of the Graduate School of the \\ University of Colorado in partial fulfillment \\ of the requirements for the degree of \\ Doctor of Philosophy \\ Department of Mathematics
}

2014 
This thesis entitled:

Projective Multiresolution Analyses over Irrational Rotation Algebras

written by Benjamin A. Purkis

has been approved for the Department of Mathematics

\begin{tabular}{c}
\hline Judith Packer \\
\hline Martin Walter
\end{tabular}

Date

The final copy of this thesis has been examined by the signatories, and we find that both the content and the form meet acceptable presentation standards of scholarly work in the above mentioned discipline. 
Purkis, Benjamin A. (Ph.D., Mathematics)

Projective Multiresolution Analyses over Irrational Rotation Algebras

Thesis directed by Prof. Judith Packer

This thesis takes the idea of projective multiresolution analyses and extends it to modules over noncommutative $\mathrm{C}^{*}$-algebras, particularly irrational rotation algebras, by constructing a concrete examples. We provide background information on irrational rotation algebras, Hilbert $\mathrm{C}^{*}$-modules, and Morita equivalence of $\mathrm{C}^{*}$-algebras. We then discuss previous definitions due to J. Packer and M. Rieffel, and modify these definitions to account for our specific situation. Then our first example of a projective multiresolution analysis over $A_{\alpha}$ is built, using the framework of a Morita equivalence due to M. Rieffel and employing a scaling function due to I. Daubechies. We then use our new PMRA to construct a module frame for a Hilbert module over an irrational rotation algebra, and using the module frame create an explicit isomorphism to a well-known Hilbert module.

We create more examples of projective multiresolution analyses over irrational rotation algebras by generalizing our construction of the initial example. We examine direct sums of PMRAs, and then show that a PMRA can be combined with a compatible Morita equivalence structure to create a new projective multiresolution analysis. This construction relies heavily on the use of balanced tensor products, and is employed to create new examples of PMRAs involving Morita equivalences due to M. Rieffel and F. Luef. 


\section{Dedication}

To my family for their unerring and constant faith, belief, and support throughout my life.

And to Jess, for always listening, encouraging, and most importantly, reminding me to smile. 


\section{Acknowledgements}

This thesis would not have been completed without the invaluable assistance of my advisor, Judy Packer. Her advice and guidance helped me to persevere. I appreciate Marty Walter for acting as my second reader and providing valuable feedback. I would also like to thank Arlan Ramsay, Frederic Latremoliere, and Karl Gustafson for serving on my committee. I would also like to acknowledge Franz Luef for fruitful conversations about my thesis.

I would be remiss if I did not thank Amy, Justin, Angela, Mike, and Katherine for being my friends throughout these seven years. I am a richer and better person from our many conversations and experiences, both mathematical and otherwise. I am particularly thankful for my fellow Horcrux hunters, Justin and Amy. Graduate school would not have been the same without the five of you. 


\section{Contents}

\section{Chapter}

1 Introduction 1

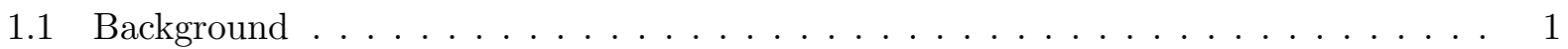

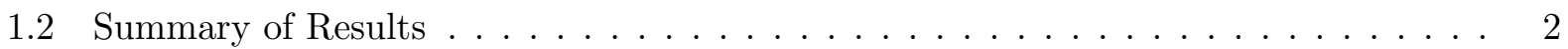

2 Preliminaries $\quad 5$

2.1 Irrational Rotation Algebras $\ldots \ldots \ldots \ldots \ldots \ldots \ldots$

2.2 Hilbert $\mathrm{C}^{*}$-modules $\ldots \ldots \ldots \ldots \ldots \ldots \ldots \ldots \ldots$

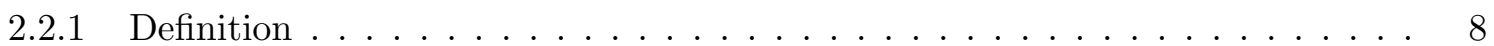

2.2 .2 Direct Sums of Hilbert Modules . . . . . . . . . . . . . . . . . . . 12

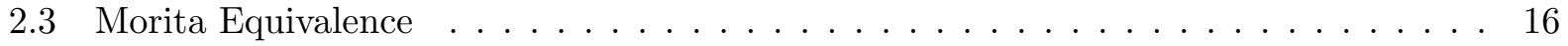

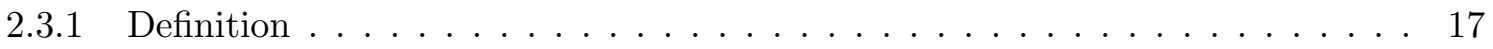

2.3 .2 Equivalence Relation . . . . . . . . . . . . . . . . . . . . 20

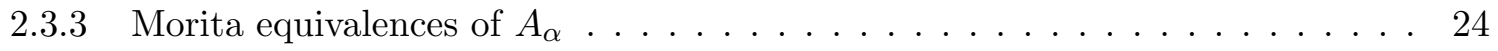

3 Projective Multiresolution Analyses 27

3.1 Defining Projective Multiresolution Analyses over $A_{\alpha} \ldots \ldots \ldots \ldots$

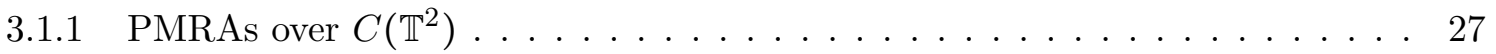

3.1.2 Projective Multiresolution Analyses over $A_{\alpha} \ldots \ldots \ldots \ldots$

3.2 A PMRA over $A_{\alpha}$ that is initially free $\ldots \ldots \ldots \ldots \ldots \ldots$

3.2 .1 The Morita Equivalence . . . . . . . . . . . . . . . . . . . . 29 
3.2 .2 The Dilation . . . . . . . . . . . . . . . . . . 30

3.2 .3 The Scaling Function . . . . . . . . . . . . . . . . . . 30

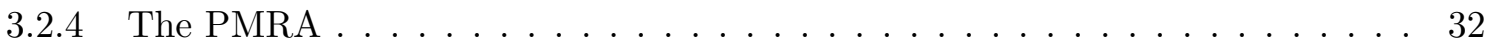

3.3 Building The Module Frame . . . . . . . . . . . . . . . . . 36

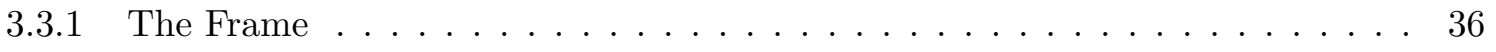

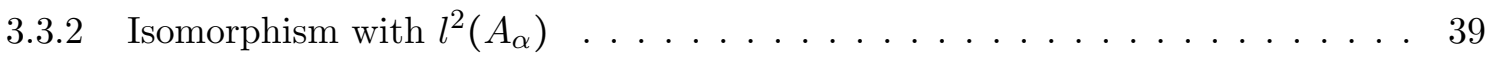

4 Other Examples of PMRAs 41

4.1 Compact Spaces $Y \ldots \ldots \ldots \ldots \ldots \ldots \ldots \ldots \ldots \ldots$

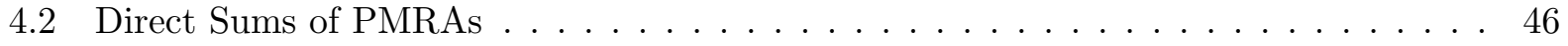

5 Tensor Products of PMRAs 49

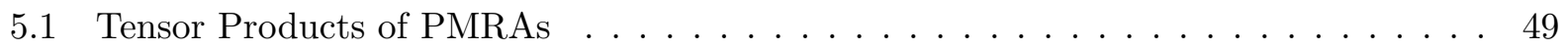

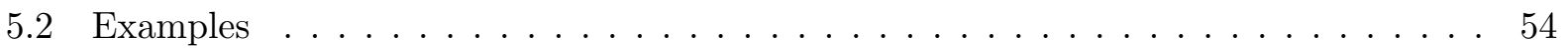

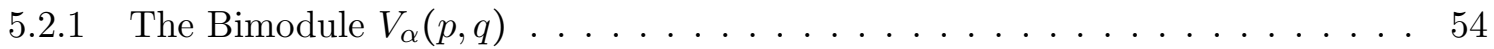

5.2 .2 Feichtinger's Algebra $S_{0}(\mathbb{R}) \ldots \ldots \ldots \ldots \ldots \ldots \ldots$

5.3 Module Frames . . . . . . . . . . . . . . . . . . . . . . 57

$\begin{array}{lr}\text { Bibliography } & 60\end{array}$ 


\section{Chapter 1}

\section{Introduction}

\section{$1.1 \quad$ Background}

Some of the more interesting objects in noncommutative harmonic analysis are the irrational rotation $C^{*}$-algebras $A_{\alpha}$. These $A_{\alpha}$ are generated by two unitaries obeying specific commutation laws, and are indexed by a single real number $\alpha$. In noncommutative geometry, irrational rotation algebras provide models for ergodic, non-transitive actions. But what makes these algebras particularly interesting is that for irrational values of $\alpha$, they are simple $C^{*}$-algebras with projections. ( $A_{\alpha}$ is not simple for rational values of $\alpha$, see [4].) In addition, for distinct irrational values of $\alpha$ in $[0,1 / 2)$, the two simple $C^{*}$-algebras are non-isomorphic, and thus irrational values of $\alpha$ index distinct isomorphism classes of simple $C^{*}$-algebras.

On the other side, commutative harmonic analysis has the geometric notion of multiresolutions, a nested system of closed subspaces in Hilbert space. Multiresolutions were first employed as a tool in the construction of new wavelets, but have also been used in noncommutative geometry and dynamical systems. These commutative and noncommutative aspects of harmonic analysis are linked by projective multiresolution analyses, as invented by J. Packer and M. Rieffel.

This paper builds upon the work done by Packer and Rieffel in [13], in the construction of projective multiresolution analyses for $L^{2}\left(\mathbb{R}^{2}\right)$. In that work, Packer and Rieffel extend the concept of multiresolution analyses and wavelet theory to work with projective modules, and in fact Hilbert $C^{*}$-modules, over $C\left(\mathbb{T}^{n}\right)$. Concrete examples of such PMRAs are constructed for the case $n=2$ where the initial module is projective yet not free. The purpose of this thesis is to explore to 
what extent this idea can be extended to Hilbert $C^{*}$-modules over noncommutative $C^{*}$-algebras, specifically irrational rotation algebras.

Interested readers can find additional background reading in the following: non-commutative geometry ([1], [6]); multiresolutions and wavelets ([11]); rotation algebras and non-commutative tori ([3], [7], [6]); and projective multiresolutions ([12], [13]).

\subsection{Summary of Results}

We begin by covering preliminary definitions for Hilbert $C^{*}$-modules, Morita equivalence of $C^{*}$-algebras, and irrational rotation algebras, specifically highlighting examples and properties that will be useful in constructing our projective multiresolution analyses. We then examine the definition and properties of the projective multiresolution analyses for $L^{2}\left(\mathbb{R}^{2}\right)$ given by Packer and Rieffel in [13]. These PMRAs employed Hilbert $C\left(\mathbb{T}^{2}\right)$-modules, and provide the groundwork for our own definition for a projective multiresolution analysis on a Hilbert $C^{\star}$-module over an irrational rotation algebra; specifically, we will make the following definition:

Definition 1.2.1. Let $\Xi$ be a countably-generated left $A_{\alpha}$-module. A projective multiresolution analysis (PMRA) for $\Xi$ over $A_{\alpha}$ is a family $\left\{V_{j}\right\}_{j \in \mathbb{Z}}$ of closed subspaces of $\Xi$ such that

(i) $V_{0}$ is a projective $A_{\alpha}$-submodule of $\Xi$,

(ii) $V_{j} \supset V_{j-1}$ for all $j$,

(iii) $\cup_{j}^{\infty} V_{j}$ is dense in $\Xi$,

(iv) $\cap_{-\infty}^{\infty} V_{j}=\{0\}$.

The first main result is the construction of an example of a PMRA over an irrational rotation algebra. We begin with a pre-equivalence bimodule from Rieffel in [16]. He shows that, for a compact space $Y$ equipped with a homeomorphism $T: Y \rightarrow Y$, the space $C_{c}(Y \times \mathbb{R})$ can be given a pre-equivalence structure for the pre- $C^{*}$-algebras $C_{c}(Y \times \mathbb{Z})$ and $C_{c}(M \times \mathbb{R})$, where $M$ is the mapping

torus for $Y$. By letting $Y=\mathbb{T}$ and $T(z)=e^{2 \pi i \alpha z}$, completing $C_{c}(Y \times \mathbb{Z})$ yields the irrational rotation 
algebra $A_{\alpha}$. The completion of $C_{c}(Y \times \mathbb{R})$, denoted $\Xi$, is then a left Hilbert $A_{\alpha}$-module, and it is that module for which we construct a projective multiresolution analysis.

A key ingredient in our construction is the use of a scaling function, $\phi$. We select this function, a function of $\mathbb{R}$ only, from the space $C_{c}(Y \times \mathbb{R})$ to satisfy the following two properties:

(1) $\int_{\mathbb{R}} \phi(r) \overline{\phi(r-n)} d r=\delta(0, n)$

(2) $\phi(r)=\sum_{j \in \mathbb{Z}} c_{j} \phi(2 r-j)$ for finitely many nonzero constants $c_{j}$

Examples of such scaling functions were constructed by Daubechies in [2]. Our initial module for the PMRA is then the action of $A_{\alpha}$ on this single scaling function, making our initial module free and singly-generated. We then apply a densely-defined dilation to create the rest of our family of subspaces, and reach the following result.

Theorem 1.2.2. The $\left\{V_{i}\right\}$ form a projective multiresolution analysis for $\Xi$ over $A_{\alpha}$.

The proof is simply to verify that the conditions of Definition 1.2.1 are satisfied. As an application, we then use the PMRA structure on $\Xi$ to construct a countable module frame for $\Xi$, which allows us to construct an explicit isomorphism from $\Xi$ to the Hilbert module $l^{2}\left(A_{\alpha}\right)$.

With one example in hand, we work to create more examples of projective multiresolution analyses. We first generalize our work from Theorem 1.2.2 using Rieffel's Morita equivalence from [16]. Instead of choosing a specific space $Y$, we allow the space to be as general as possible; namely, $Y$ must be a compact space equipped with a finite, invariant measure $\nu$. Under these hypotheses, we can construct in a completely similar fashion a PMRA for the equivalence bimodule, which is now a $C(Y) \times \mathbb{Z}$-module.

The remainder of the thesis examines ways in which existing projective multiresolution analyses can be combined with other items to yield new PMRA structures. It is known that the direct sum of two Hilbert modules (over the same $C^{*}$-algebra) is again a Hilbert module; if these Hilbert modules both have compatible PMRA structures associated with them, then their direct sum also has a PMRA structure, as seen in our next result. 
Theorem 1.2.3. Let $A$ be a $C^{*}$-algebra, and suppose that $\Xi$ and $\Omega$ are both left (or right) Hilbert A-modules. Suppose further that the families of subspaces $\left\{V_{i}\right\}$ and $\left\{W_{i}\right\}$ are PMRAs for $\Xi$ and $\Omega$, respectively. Then the family of subspaces $\left\{Y_{i}\right\}$ given by $Y_{i}=V_{i} \oplus W_{i}$ forms a left (or right) A-PMRA structure for the Hilbert module $\Xi \oplus \Omega$.

To this point, all of the PMRAs constructed have had initial modules that were free. In order to create examples of PMRAs in which the initial module is not free, we turn to (balanced) tensor products. Our inspiration comes from the construction of the internal tensor product of two equivalence bimodules as follows: if $X$ is an $A-B$ equivalence bimodule and $Y$ is a $B-C$ equivalence bimodule, then the balanced tensor product $X \otimes_{B} Y$ forms a pre-equivalence bimodule for $A$ and $C$. If the bimodule $Y$ has a left $B$-PMRA structure associated to it, then our main result states that the balanced tensor product also has a PMRA structure.

Theorem 1.2.4. Suppose $X$ is an $A-B$ equivalence bimodule, and $Y$ is a $B-C$ equivalence bimodule. Further suppose that $Y$ has a left B-PMRA structure $\left\{V_{i}\right\}$. Then there exists a left A-PMRA structure for the bimodule $X \otimes_{B} Y$.

This theorem is proven by constructing the new projective multiresolution analysis from the original structure and then verifying that the conditions of Definition 1.2.1. We finish by applying the theorem to create two examples involving the bimodules $V_{\alpha}(p, q)$ of Rieffel ([18]) and Feichtinger's algebra $S_{0}(\mathbb{R})$ as seen in Luef ([10]). 


\section{Chapter 2}

\section{Preliminaries}

Projective multiresolution analyses are constructed out of Hilbert modules, which are an extension of the idea of a Hilbert space. Since we wish to create examples of these involving noncommutative $\mathrm{C}^{*}$-algebras, we will also consider irrational rotation algebras, which are in some senses the simplest noncommutative $\mathrm{C}^{*}$-algebra. Some of our examples and constructions will also be informed by the idea of Morita equivalence of $\mathrm{C}^{*}$-algebras, so we will delve into that area as well.

The definitions and examples that follow will not only give the necessary background, but also serve to fix our notations and conventions. Only the basics will be covered here; for more details, we refer the reader to [9], [15], and [16].

\subsection{Irrational Rotation Algebras}

Irrational rotation algebras arise in many different places in mathematics, and thus can be defined in several ways. We will define them in terms a universal property, and then discuss useful alternate characterizations.

Definition 2.1.1. Let $\alpha$ be an irrational number between 0 and 1 . The irrational rotation algebra $A_{\alpha}$ is the universal $\mathrm{C}^{*}$-algebra generated by two unitaries, $U$ and $V$, satisfying the commutation relation

$$
U V=e^{2 \pi i \alpha} V U
$$


The idea behind an irrational rotation algebra is rotations of the unit circle, $\mathbb{T}$. We can directly realize $A_{\alpha}$ by letting $z(t)=e^{2 \pi i t}$ and $H=L^{2}(\mathbb{T})$ be a Hilbert space. Then define two unitary operators on $H$ :

$$
U f(t)=z(t) f(t) \quad \text { and } \quad V f(t)=f(t-\alpha)
$$

Here $f(t) \in H=L^{2}(\mathbb{T}), U$ is playing the role of modulation, and $V$ is rotation of the circle by $2 \pi \alpha$. A straightforward calculation then shows that:

$$
\begin{gathered}
V U f(t)=U f(t-\alpha)=z(t-\alpha) f(t-\alpha)=e^{-2 \pi i \alpha} z(t) V f(t)=e^{-2 \pi i \alpha} U V f(t) \\
\Longrightarrow U V=e^{2 \pi i \alpha} V U .
\end{gathered}
$$

Remark 2.1.2. Suppose $U^{\prime}$ and $V^{\prime}$ are unitaries satisfying Equation 2.1 , and denote by $C^{*}\left(U^{\prime}, V^{\prime}\right)$ the $\mathrm{C}^{*}$-algebra generated by these two unitaries. The universal property in Definition 2.1.1 means there is a homomorphism $\phi: A_{\alpha} \rightarrow C^{*}\left(U^{\prime}, V^{\prime}\right)$ that maps $U \rightarrow U^{\prime}$ and $V \rightarrow V^{\prime}$; in fact, it can be shown that $\phi$ is an isomorphism, so that this $\mathrm{C}^{*}$-algebra is unique up to isomorphism.

There is one representation of an irrational rotation algebra that we will be particularly interested in, as it allows us to explicitly characterize a dense subset of $A_{\alpha}$.

Consider $S$ to be the rotation of the unit circle, $\mathbb{T}$, by an angle of $2 \pi \alpha$. If $C(\mathbb{T})$ denotes the continuous functions on the unit circle, then $S$ can also be viewed as acting on $C(\mathbb{T})$. This induces an action of $\mathbb{Z}$ on $C(\mathbb{T})$ through powers of $S$, and by forming the cross product algebra $C(\mathbb{T}) \times_{\alpha} \mathbb{Z}$ for this action, we obtain $A_{\alpha}$.

With this setup, we can realize a dense subset of $A_{\alpha}$ as

$$
f(t, n)=\sum_{F \subset \mathbb{Z}} f_{n}(t) U^{n}
$$

for $F$ a finite subset of $\mathbb{Z}$ and $f_{n} \in C(\mathbb{T})$. Here the unitary $V$ is represented by

$$
f_{V}(t, n)= \begin{cases}e^{2 \pi i t} & n=0 \\ 0 & n \neq 0\end{cases}
$$


and the norm on such finite sums is obtained from any suitable representation on $L^{2}(\mathbb{T})$.

The specific value of $\alpha$ changes the structure of $A_{\alpha}$ significantly. In particular, rational values of $\alpha$ yield very different algebras, known as rational rotation algebras. But even among irrational $\alpha$, the $\mathrm{C}^{*}$-algebras involved are rarely isomorphic. The question of when two irrational rotation algebras are isomorphic was studied by Rieffel in [17], who showed the following theorem using projections of specific trace:

Proposition 2.1.3. [17] Suppose $\alpha$ and $\beta$ are irrational numbers between 0 and 1 . If $A_{\alpha}$ is isomorphic to $A_{\beta}$, then one of the following occurs:

(1) $\beta=\alpha$

(2) $\beta=1-\alpha$

Remark 2.1.4. An irrational rotation algebra is really a special case of a noncommutative $2 d$ torus, which is generated by $2 d$ unitaries satisfying similar commutation relations with rotation values from a real-valued, skew-symmetric matrix. In this thesis we will restrict our attention to 2-tori, or irrational rotation algebras.

\section{$2.2 \quad$ Hilbert $\mathrm{C}^{*}$-modules}

The theory of Hilbert modules has its roots in a paper of Kaplansky [8], and was put into a general framework by Paschke in 1973([14]). Marc Rieffel then used Hilbert modules to motivate his idea of strong Morita equivalence of $\mathrm{C}^{*}$-algebras. We will be interested both in Hilbert modules for Morita equivalence and as interesting objects in their own right.

Hilbert modules are an extension of the idea of Hilbert spaces, with the field of scalars replaced by a $\mathrm{C}^{*}$-algebra. Hence they have an inner product that takes its values in a $\mathrm{C}^{*}$-algebra, and a norm induced by that inner product for which the module is complete.

The background here is restricted to relevant information, and appears in [9] and [15]. For a fuller treatment, see those references. 


\subsubsection{Definition}

We begin by defining an inner product module.

Definition 2.2.1. [15] Given a $C^{*}$-algebra $A$, an inner product $A$-module $X$ is an $A$-module equipped with an $A$-valued inner product $\langle\cdot, \cdot\rangle_{A}$ satisfying

(i) $\langle x, \lambda y+\mu z\rangle_{A}=\lambda\langle x, y\rangle_{A}+\mu\langle x, z\rangle_{A}$

(ii) $\langle x, y \cdot a\rangle=\langle x, y\rangle_{A} a$

(iii) $\langle x, y\rangle_{A}^{*}=\langle y, x\rangle_{A}$

(iv) $\langle x, x\rangle_{A} \geq 0$ as an element of $A$

(v) $\langle x, x\rangle_{A}=0$ implies that $x=0$

Remark 2.2.2. The conditions on the inner product above are written for a right inner product $A$-module; there are analogous conditions for a left inner product $A$-module.

It is important to note that by replacing a field of scalars with the $\mathrm{C}^{*}$-algebra $A$, we have an inner product which takes its values in the $\mathrm{C}^{*}$-algebra $A$. We can treat these inner products similarly to our usual inner products just with slightly more nuance. For example, we have a version of Cauchy-Schwartz for such inner products.

Lemma 2.2.3 (Cauchy-Schwartz). Let $X$ be an inner product $A$-module for some $C^{*}$-algebra $A$. Then for all $x, y \in X$,

$$
\langle x, y\rangle_{A}^{*}\langle x, y\rangle_{A} \leq\left\|\langle x, x\rangle_{A}\right\|\langle y, y\rangle_{A}
$$

as elements of $A$.

Proof. We will show that $\left\|\langle x, x\rangle_{A}\right\|\langle y, y\rangle_{A}-\langle x, y\rangle_{A}^{*}\langle x, y\rangle_{A}$ is a positive element of $A$; to do this, we will use the fact that an element of a $\mathrm{C}^{*}$-algebra is positive if $\rho(a) \geq 0$ for all states $\rho$ of $A$, and show that for every such state,

$$
\rho\left(\left\|\langle x, x\rangle_{A}\right\|\langle y, y\rangle_{A}-\langle x, y\rangle_{A}^{*}\langle x, y\rangle_{A}\right) \geq 0 .
$$


Let $\rho$ be a state of $A$. The map $(w, z) \mapsto \rho\left(\langle w, z\rangle_{A}\right)$ is then a positive sesquilinear form on $X$, and we can apply the ordinary Cauchy-Schwartz inequality to obtain

$$
\left|\rho\left(\langle w, z\rangle_{A}\right)\right| \leq \rho\left(\langle w, w\rangle_{A}\right)^{\frac{1}{2}} \rho\left(\langle z, z\rangle_{A}\right)^{\frac{1}{2}}
$$

Using this fact with $w=x\langle x, y\rangle_{A}$ and $z=y$, we calculate:

$$
\begin{aligned}
& \rho\left(\langle x, y\rangle_{A}^{*}\langle x, y\rangle_{A}\right)=\rho\left(\left\langle x\langle x, y\rangle_{A}, y\right\rangle_{A}\right) \\
\leq & \rho\left(\left\langle x\langle x, y\rangle_{A}, x\langle x, y\rangle_{A}\right\rangle_{A}\right)^{\frac{1}{2}} \rho\left(\langle y, y\rangle_{A}\right)^{\frac{1}{2}} \\
= & \rho\left(\langle x, y\rangle_{A}^{*}\langle x, x\rangle_{A}\langle x, y\rangle_{A}\right)^{\frac{1}{2}} \rho\left(\langle y, y\rangle_{A}\right)^{\frac{1}{2}}
\end{aligned}
$$

We now use an inequality in $\mathrm{C}^{*}$-algebras: $b^{*} c b \leq\|c\| b^{*} b$ for any $b$ and positive $c$. We use $b=\langle x, y\rangle_{A}$ and $c=\langle x, x\rangle_{A} \geq 0$ (since $X$ is an inner product module) to conclude that

$$
\rho\left(\langle x, y\rangle_{A}^{*}\langle x, y\rangle_{A}\right) \leq\left(\left\|\langle x, x\rangle_{A}\right\| \rho\left(\langle x, y\rangle_{A}^{*}\langle x, y\rangle_{A}\right)\right)^{\frac{1}{2}} \rho\left(\langle y, y\rangle_{A}\right)^{\frac{1}{2}} .
$$

Squaring both sides, canceling a common $\rho\left(\langle x, y\rangle_{A}^{*}\langle x, y\rangle_{A}\right)$, and rearranging gives us

$$
\rho\left(\left\|\langle x, x\rangle_{A}\right\|\langle y, y\rangle_{A}-\langle x, y\rangle_{A}^{*}\langle x, y\rangle_{A}\right) \geq 0
$$

Since $\rho$ was an arbitrary state, we have shown this to be true for all states $\rho$, and we have our lemma.

We can also use $\mathrm{C}^{*}$-algebra valued inner products to induce norms on inner product modules, analogously to inner product spaces.

Proposition 2.2.4. Given an inner product $A$-module $X$,

$$
\|x\|_{A}:=\left\|\langle x, x\rangle_{A}\right\|^{\frac{1}{2}}
$$

defines a norm on $X$. 
Proof. This follows from a few straightforward calculations. First, for $\lambda \in \mathbb{C}$ and $x \in X$ :

$$
\|\lambda x\|_{A}^{2}=\left\|\langle\lambda x, \lambda x\rangle_{A}\right\|=\lambda^{2}\left\|\langle x, x\rangle_{A}\right\|=\lambda^{2}\|x\|_{A}^{2}
$$

from which we get that $\|\lambda x\|_{A}=|\lambda|\|x\|_{A}$.

Condition (iv) of the inner product module definition implies that $\|x\|_{A}=\left\|\langle x, x\rangle_{A}\right\|^{\frac{1}{2}} \geq 0$, and condition (v) gives that $\|x\|_{A}=0 \Longrightarrow x=0$.

Lastly, Equation 2.2 gives that $\left\|\langle x, y\rangle_{A}\right\| \leq\|x\|_{A}\|y\|_{A}$, so that

$$
\begin{gathered}
\|x+y\|_{A}^{2} \leq\left\|\langle x, x\rangle_{A}\right\|+\left\|\langle x, y\rangle_{A}\right\|+\left\|\langle y, x\rangle_{A}\right\|+\left\|\langle y, y\rangle_{A}\right\| \\
\leq\|x\|_{A}^{2}+2\|x\|_{A}\|y\|_{A}+\|y\|_{A}^{2} \\
=\left(\|x\|_{A}+\|y\|_{A}\right)^{2}
\end{gathered}
$$

and taking square roots gives $\|x+y\|_{A} \leq\|x\|_{A}+\|y\|_{A}$.

Definition 2.2.5. Given a $\mathrm{C}^{*}$-algebra $A$, a Hilbert $A$-module is an inner product $A$-module which is complete in the norm $\|\cdot\|_{A}$ defined above. A Hilbert $A$-module is said to be full if

$$
\operatorname{span}\left\{\langle x, y\rangle_{A}: x, y \in X\right\}
$$

is dense in the $\mathrm{C}^{*}$-algebra $A$.

Example 2.2.6. Given any $\mathrm{C}^{*}$-algebra $A$, we can make $A$ into a full (right) Hilbert $A$-module by letting the action be right multiplication in $A$ and the inner product be given by $\langle a, b\rangle_{A}=a^{\star} b$.

Proof. The calculations are straightforward:

(i) $\langle a, \lambda b+\mu c\rangle_{A}=a^{*}(\lambda b+\mu c)=\lambda a^{*} b+\mu a^{*} c=\lambda\langle a, b\rangle_{A}+\mu\langle a, c\rangle_{A}$

(ii) $\langle a, b \cdot c\rangle_{A}=a^{*}(b c)=\left(a^{*} b\right) c=\langle a, b\rangle_{A} c$

(iii) $\langle a, b\rangle_{A}^{*}=\left(a^{*} b\right)^{*}=b^{*} a=\langle b, a\rangle_{A}$ 
(iv) For any $a \in A,\langle a, a\rangle_{A}=a^{*} a \geq 0$.

(v) $\langle a, a\rangle_{A}=0 \Longrightarrow a^{*} a=0 \Longrightarrow\left\|a^{*} a\right\|=0 \Longrightarrow\|a\|^{2}=0 \Longrightarrow a=0$

The norm induced by this inner product actually coincides with the usual norm on the $\mathrm{C}^{*}$ algebra:

$$
\|a\|_{A}^{2}=\left\|\langle a, a\rangle_{A}\right\|=\left\|a^{*} a\right\|=\|a\|^{2}
$$

and therefore $A$ is complete in the norm $\|\cdot\|_{A}$, and is a Hilbert $A$-module. It is a full Hilbert $A$-module because products of the form $a^{\star} b$ are dense in $A$.

Thus far, the definition of a Hilbert module has been analogous to that of a Hilbert space. If this is truly to be a generalization of Hilbert spaces, however, we must be able to get the traditional Hilbert spaces from this construction. The next example shows that, with a careful choice of inner product, we can do so.

Example 2.2.7. Let $X$ be a right inner product $\mathbb{C}$-module with the inner product

$$
\langle h, k\rangle_{\mathbb{C}}=(k \mid h)
$$

for all $h, k \in \mathbb{C}$, where $(\cdot \cdot)$ denotes the usual inner product of complex numbers. Then $X$ is a Hilbert $\mathbb{C}$-module, and in fact is also a Hilbert space.

Remark 2.2.8. When defining the inner product, we swap the order of variables to align with conventions. When working with right Hilbert modules, it is customary and convenient to work with inner products which are conjugate-linear in the first variable. The convention with Hilbert spaces, however, is for the inner products to be conjugate linear in the second variable. The swap in order preserves both conventions without difficulty.

Hilbert modules share many of the properties that Hilbert spaces have, but not all of them. For instance, not all bounded, $A$-linear operators between Hilbert modules are adjointable, as seen in the following example. 
Example 2.2.9. Let $D$ be the closed unit disk, and let $\partial D$ be the boundary of $D$, i.e. the unit circle. Let $F$ be the Hilbert module $C(D)$, continuous functions on the disk, and let

$$
E=\{f \in F: f(\partial D)=\{0\}\} .
$$

Both $E$ and $F$ are Hilbert $C(D)$-modules. Consider the inclusion map $i: E \rightarrow F$; if this map had an adjoint, $i^{\star}$, it would have to satisfy $i^{\star}(1)=1$. But $1 \notin E$, and thus $i$ cannot be adjointable.

\subsubsection{Direct Sums of Hilbert Modules}

In later work, we will be interested in the direct sum of Hilbert modules. Here we will show that the direct sum of two $A$-modules is again an $A$-modules, and then examine a specific case of infinite direct sum that will resurface later.

Proposition 2.2.10 (Direct Sum of Hilbert Modules). Let $X$ and $Y$ be right A-modules for $a$ $C^{*}$-algebra A. Then

$$
Z=X \oplus Y:=\{(x, y): x \in X \text { and } y \in Y\}
$$

is a Hilbert A-module under the inner product

$$
\left\langle(x, y),\left(x_{1}, y_{1}\right)\right\rangle_{A}:=\left\langle x, x_{1}\right\rangle_{A}+\left\langle y, y_{1}\right\rangle_{A}
$$

Proof. We first verify the conditions of an inner product $A$-module, and then show completeness under the norm. For the inner product conditions:

(i) $\left\langle(x, y), \lambda\left(x_{1}, y_{1}\right)+\mu\left(x_{2}, y_{2}\right)\right\rangle_{A}=\left\langle x, \lambda x_{1}+\mu x_{2}\right\rangle_{A}+\left\langle y, \lambda y_{1}+\mu y_{2}\right\rangle_{A}$

$$
\begin{gathered}
=\lambda\left\langle x, x_{1}\right\rangle_{A}+\mu\left\langle x, x_{2}\right\rangle_{A}+\lambda\left\langle y, y_{1}\right\rangle_{A}+\mu\left\langle y, y_{2}\right\rangle_{A} \\
=\lambda\left(\left\langle x, x_{1}\right\rangle_{A}+\left\langle y, y_{1}\right\rangle_{A}\right)+\mu\left(\left\langle x, x_{2}\right\rangle_{A}+\left\langle y, y_{2}\right\rangle_{A}\right) \\
=\lambda\left\langle(x, y),\left(x_{1}, y_{1}\right)\right\rangle_{A}+\mu\left\langle(x, y),\left(x_{2}, y_{2}\right)\right\rangle_{A}
\end{gathered}
$$

(ii) $\left\langle(x, y),\left(x_{1}, y_{1}\right) \cdot a\right\rangle_{A}=\left\langle x, x_{1} \cdot a\right\rangle_{A}+\left\langle y, y_{1} \cdot a\right\rangle_{A}$

$$
=\left\langle x, x_{1}\right\rangle_{A} a+\left\langle y, y_{1}\right\rangle_{A} a=\left\langle(x, y),\left(x_{1}, y_{1}\right)\right\rangle_{A} a
$$


(iii) $\left\langle(x, y),\left(x_{1}, y_{1}\right)\right\rangle_{A}^{*}=\left\langle x, x_{1}\right\rangle_{A}^{*}+\left\langle y, y_{1}\right\rangle_{A}^{*}=\left\langle x_{1}, x\right\rangle_{A}+\left\langle y_{1}, y\right\rangle_{A}=\left\langle\left(x_{1}, y_{1}\right),(x, y)\right\rangle_{A}$

(iv) $\langle(x, y),(x, y)\rangle_{A}=\langle x, x\rangle_{A}+\langle y, y\rangle_{A} \geq 0$, since both $X$ and $Y$ satisfy condition (iv).

(v) $\langle(x, y),(x, y)\rangle_{A}=0 \Longrightarrow\langle x, x\rangle_{A}+\langle y, y\rangle_{A}=0$; since both $\langle x, x\rangle_{A}$ and $\langle y, y\rangle_{A}$ are nonnegative, they must both be 0 for this equation to hold. But then $x=0$ and $y=0$, since $X$ and $Y$ satisfy condition (v).

Hence $Z$ is an inner product $A$-module. To show completeness, we note that

$$
\|x\|_{A}^{2}=\left\|\langle x, x\rangle_{A}\right\| \leq\left\|\langle x, x\rangle_{A}+\langle y, y\rangle_{A}\right\|=\|(x, y)\|_{A}^{2} \leq\|x\|_{A}^{2}+\|y\|_{A}^{2}
$$

and taking square roots gives us

$$
\|x\|_{A} \leq\|(x, y)\|_{A} \leq \sqrt{\|x\|_{A}^{2}+\|y\|_{A}^{2}} .
$$

Similarly,

$$
\|y\|_{A} \leq\|(x, y)\|_{A} \leq \sqrt{\|x\|_{A}^{2}+\|y\|_{A}^{2}}
$$

so that

$$
\max \left\{\|x\|_{A},\|y\|_{A}\right\} \leq\|(x, y)\|_{A} \leq \sqrt{\|x\|_{A}^{2}+\|y\|_{A}^{2}}
$$

and then the completeness of $Z$ follows from the completeness of $X$ and $Y$.

Remark 2.2.11. This construction naturally extends to finite direct sums, in particular to $n$ copies of a Hilbert module $X$, which is denoted $X^{n}$.

Infinite direct sums of Hilbert modules can also be formed; a general treatment of this can be found in [9]. For the purposes of this thesis, we will be content to restrict our attention to a specific example, taken from [15].

Example 2.2.12. [15] Let $A$ be a $\mathrm{C}^{*}$-algebra, and define

$$
l^{2}(A)=\left\{\left(a_{i}\right): a_{i} \in A \text { and } \sum_{i=1}^{\infty}\left\|a_{i}\right\|^{2}<\infty\right\}
$$


Then $l^{2}(A)$ is a (right) Hilbert $A$-module under the action and inner product given by:

$$
\left(a_{i}\right) \cdot a:=\left(a_{i} a\right) \quad\left\langle\left(a_{i}\right),\left(b_{i}\right)\right\rangle_{A}:=\sum_{i=1}^{\infty} a_{i}^{*} b_{i}
$$

Proof. The first step is to show that $\left(a_{i}\right) \cdot a \in l^{2}(A)$ and $\left\langle\left(a_{i}\right),\left(b_{i}\right)\right\rangle_{A}$ converges in $A$. For the first, choose $M<N$; we consider the partial sums of $\sum_{i=1}^{\infty}\left(a_{i} a\right)^{*} a_{i} a$ :

$$
\sum_{i=M}^{N}\left(a_{i} a\right)^{*} a_{i} a=a^{*}\left(\sum_{i=M}^{N} a_{i}^{*} a_{i}\right) a \leq\left\|\sum_{i=M}^{N} a_{i}^{*} a_{i}\right\| a^{*} a
$$

Since $\left(a_{i}\right) \in l^{2}(A)$, we know that the $\sum_{i=M}^{N} a_{i}^{*} a_{i}$ are Cauchy; hence the sum $\sum_{i=1}^{\infty}\left(a_{i} a\right)^{*} a_{i} a$ converges in $A$, and therefore $\left(a_{i}\right) \cdot a$ is in $l^{2}(A)$ as desired.

For the inner product, again choose $M<N$ and consider the partial sums of the defined inner product, for which we will need the following lemma:

\section{Lemma 2.2.13.}

$$
\left\|\sum_{i=1}^{n} a_{i}^{*} b_{i}\right\|^{2} \leq\left\|\sum_{i=1}^{n} a_{i}^{*} a_{i}\right\|\left\|\sum_{i=1}^{n} b_{i}^{*} b_{i}\right\|
$$

Proof. This is a consequence of the Cauchy-Schwartz inequality (Equation 2.2), applied to a finite direct sum $\oplus_{i=1}^{n} A$, where $A$ is the Hilbert module from Example 2.2.6. Taking the norm of both sides of Equation 2.2 gives

$$
\left\|\langle x, y\rangle_{A}\right\|^{2} \leq\left\|\langle x, x\rangle_{A}\right\|\left\|\langle y, y\rangle_{A}\right\|
$$

We now apply this to the inner product of $\bigoplus_{i=1}^{n} A$, and use the formula of the inner product on $A$ to give:

$$
\left\|\sum_{i=1}^{n} a_{i}^{\star} b_{i}\right\|^{2} \leq\left\|\sum_{i=1}^{n} a_{i}^{*} a_{i}\right\|\left\|\sum_{i=1}^{n} b_{i}^{\star} b_{i}\right\|
$$

as desired.

Now using the lemma, we set $n=N-M$ and shift the index on the sums to get

$$
\left\|\sum_{i=M}^{N} a_{i}^{*} b_{i}\right\|^{2} \leq\left\|\sum_{i=M}^{N} a_{i}^{*} a_{i}\right\|\left\|\sum_{i=M}^{N} b_{i}^{*} b_{i}\right\|
$$

Since the sums of the $a_{i}^{*} a_{i}$ and $b_{i}^{*} b_{i}$ converge in $A$, the convergence of the inner product follows. We now verify that $l^{2}(A)$ is an inner product $A$-module; the calculations are straightforward: 
(i) $\left\langle\left(a_{i}\right), \lambda\left(b_{i}\right)+\mu\left(c_{i}\right)\right\rangle_{A}=\sum_{i=1}^{\infty} a_{i}^{*}\left(\lambda b_{i}+\mu c_{i}\right)=\lambda \sum_{i=1}^{\infty} a_{i}^{*} b_{i}+\mu \sum_{i=1}^{\infty} a_{i}^{*} c_{i}$

$$
=\lambda\left\langle\left(a_{i}\right),\left(b_{i}\right)\right\rangle_{A}+\mu\left\langle\left(a_{i}\right),\left(c_{i}\right)\right\rangle_{A}
$$

(ii) $\left\langle\left(a_{i}\right),\left(b_{i}\right) \cdot a\right\rangle_{A}=\sum_{i=1}^{\infty} a_{i}^{*}\left(b_{i} a\right)=\left(\sum_{i=1}^{\infty} a_{i}^{*} b_{i}\right) a=\left\langle\left(a_{i}\right),\left(b_{i}\right)\right\rangle_{A} a$

(iii) $\left\langle\left(a_{i}\right),\left(b_{i}\right)\right\rangle_{A}^{*}=\left(\sum_{i=1}^{\infty} a_{i}^{*} b_{i}\right)^{*}=\sum_{i=1}^{\infty} b_{i}^{*} a_{i}=\left\langle\left(b_{i}\right),\left(a_{i}\right)\right\rangle_{A}$

(iv) $\left\langle\left(a_{i}\right),\left(a_{i}\right)\right\rangle_{A}=\sum_{i=1}^{\infty} a_{i}^{*} a_{i}$, and since $a_{i}^{*} a_{i} \geq 0$ for all $i$, condition (iv) follows.

(v) $\left\langle\left(a_{i}\right),\left(a_{i}\right)\right\rangle_{A}=0 \Longrightarrow \sum_{i=1}^{\infty} a_{i}^{*} a_{i}=0$, and since $a_{i}^{*} a_{i} \geq 0$ for all $i$, this forces $a_{i}=0$ for all $i$, and hence $\left(a_{i}\right)=0$.

Thus $l^{2}(A)$ is an inner product $A$-module, and it remains only to show that it is complete under the norm induced by the inner product.

Suppose we have a sequence $\left\{\left(x_{i}^{n}\right)\right\}$ of elements of $l^{2}(A)$ that is Cauchy. For a fixed $n$, we have that $\left\|x_{i}^{n}\right\|_{A} \leq\left\|\left(x_{i}^{n}\right)\right\|_{A}$, i.e. the norm of an element is smaller than the norm of the sequence. Therefore for any fixed $n$, the sequence $\left\{x_{i}^{n}\right\}_{i}$ is a Cauchy sequence in the $\mathrm{C}^{*}$-algebra $A$, and therefore converges to some element $a_{i}$ in $A$. Our goal is then to show that $\left(a_{i}\right) \in l^{2}(A)$ and furthermore, that $\left\{\left(x_{i}^{n}\right)\right\}$ converges to $\left(a_{i}\right)$ in $l^{2}(A)$.

Given any sequence $\left(b_{i}\right)$ of elements of $A$, and for $n>m$, we define

$$
\left\|\left(b_{i}\right)\right\|_{m, n}:=\left\|\sum_{i=m}^{n} b_{i}^{\star} b_{i}\right\|^{\frac{1}{2}}
$$

and note that if $\left(b_{i}\right) \in l^{2}(A)$, then $\left\|\left(b_{i}\right)\right\|_{m, n} \leq\left\|\left(b_{i}\right)\right\|_{A}$ for any choice of $n>m$.

Let $\epsilon>0$. Because the initial sequence $\left\{x_{i}^{n}\right\}$ is Cauchy, there is an $N$ such that $\left\|\left(x_{i}^{k}\right)-\left(x_{i}^{l}\right)\right\|_{A}<$ $\frac{\epsilon}{3}$ when $k, l \geq N$. In addition, we may choose a $P \geq N$ so that

$$
\left\|\sum_{i=P}^{\infty}\left(x_{i}^{N}\right)^{*} x_{i}^{N}\right\|^{\frac{1}{2}}<\frac{\epsilon}{3}
$$

Now fix $m, n \geq P$, and find an $M \geq N$ such that $\left\|\left(a_{i}\right)-\left(x_{i}^{M}\right)\right\|_{m, n}<\frac{\epsilon}{3}$. This is possible because the $\mathrm{m}, \mathrm{n}$ norm only involves finitely many entries. 
We now calculate $\left\|\left(a_{i}\right)\right\|_{m, n}=\left(\sum_{i=m}^{n} a_{i}^{*} a_{i}\right)^{\frac{1}{2}}$; since these are the partial sums of $\sum_{i=1}^{\infty} a_{i}^{\star} a_{i}$, bounding them by $\epsilon$ will show that $\left(a_{i}\right) \in l^{2}(A)$ :

$$
\begin{gathered}
\left\|\left(a_{i}\right)\right\|_{m, n} \leq\left\|\left(a_{i}\right)-\left(x_{i}^{M}\right)\right\|_{m, n}+\left\|\left(x_{i}^{M}\right)-\left(x_{i}^{N}\right)\right\|_{m, n}+\left\|\left(x_{i}^{N}\right)\right\|_{m, n} \\
\quad \leq\left\|\left(a_{i}\right)-\left(x_{i}^{M}\right)\right\|_{m, n}+\left\|\left(x_{i}^{M}\right)-\left(x_{i}^{N}\right)\right\|_{A}+\left\|\sum_{i=P}^{\infty}\left(x_{i}^{N}\right)^{*} x_{i}^{N}\right\|^{\frac{1}{2}} \leq \epsilon
\end{gathered}
$$

Therefore, $\left(a_{i}\right) \in l^{2}(A)$. We now show that $\left\{\left(x_{i}^{n}\right)\right\}$ converges to $\left(a_{i}\right)$; let $\epsilon>0$. Then there is an $N$ such that $\left\|\left(x_{i}^{n}\right)-\left(x_{i}^{m}\right)\right\|_{A}<\epsilon$ whenever $n, m \geq N$. If we square both sides and take the $k$-th partial sum, we get

$$
\left\|\sum_{i=1}^{k}\left(x_{i}^{n}-x_{i}^{m}\right)^{*}\left(x_{i}^{n}-x_{i}^{m}\right)\right\| \leq \epsilon^{2}
$$

which allows us to let $m \rightarrow \infty$ and obtain

$$
\left\|\sum_{i=1}^{k}\left(x_{i}^{n}-a_{i}\right)^{*}\left(x_{i}^{n}-a_{i}\right)\right\| \leq \epsilon^{2} .
$$

This is valid for all $n \geq N$ and all $k$, and since $\left(x_{i}^{n}\right)-\left(a_{i}\right) \epsilon l^{2}(A)$, we get $\left\|\left(x_{i}^{n}\right)-\left(a_{i}\right)\right\|_{A} \leq \epsilon$ for $n \geq N$. Hence $\left\{\left(x_{i}^{n}\right)\right\}$ converges to $\left(a_{i}\right)$, and $l^{2}(A)$ is complete and hence a Hilbert $A$-module.

Remark 2.2.14. The notation $l^{2}(A)$ is used to highlight the analogy with the usual Hilbert space $l^{2}$; other notations are used, including $\oplus A$ and $\mathrm{H}_{A}$.

\subsection{Morita Equivalence}

Morita equivalence first arose in the area of ring theory. Rings that are Morita equivalent have naturally equivalent categories of modules, and the Morita Theorem says that in this case, there is a bimodule that implements this equivalence. In the 1970s, Marc Rieffel wrote a series of papers in which he outlined a useful form of Morita equivalence for $\mathrm{C}^{*}$-algebras that he termed strong Morita equivalence. Since we are not interested in the ring theory case of Morita equivalence, we will drop the term 'strong' from Rieffel's terminology. Here we will outline the definition and some of the basic properties of Morita equivalence. 


\subsubsection{Definition}

Morita equivalence forms an equivalence relationship among $\mathrm{C}^{*}$-algebras, and will form a basis for our example of the PMRA.

Definition 2.3.1. [15] Two $C^{*}$-algebras $A$ and $B$ are said to be Morita equivalent if there exists an $A$ - $B$ bimodule $X$ such that

(i) $X$ is a full left Hilbert $A$-module, and a full right Hilbert $B$-module,

(ii) for all $x, y \in X, a \in A, b \in B$,

$$
\langle a \cdot x, y\rangle_{B}=\left\langle x, a^{*} \cdot y\right\rangle_{B} \text { and }{ }_{A}\langle x \cdot b, y\rangle={ }_{A}\left\langle x, y \cdot b^{*}\right\rangle,
$$

(iii) for all $x, y, z \in X$,

$$
{ }_{A}\langle x, y\rangle \cdot z=x \cdot\langle y, z\rangle_{B} .
$$

The bimodule $X$ in the above definition is known as an equivalence bimodule, and is sometimes said to implement the Morita equivalence of $A$ and $B$. Equation 2.5 is an associativity condition that assures the inner products behave well with respect to each other; it is sometimes known as the Rieffel associativity condition.

Equation 2.4 of the definition says that $A$ acts by adjointable operators on $X$ as a right $B$-module, while $B$ acts by adjointable operators on $X$ as a left $A$-module. This condition can actually be replaced by an equivalent condition, which we will state but not prove here; see [15] for the full argument.

Proposition 2.3.2. Let $A$ and $B$ be $C^{*}$-algebras and suppose that $X$ is an $A-B$ bimodule satisfying conditions (i) and (iii) of Definition 2.3.1. Then $X$ is an equivalence bimodule if and only if $X$ satisfies

(ii) for all $a \in A, b \in B$, and $x \in X$,

$$
\langle a \cdot x, a \cdot x\rangle_{B} \leq\|a\|^{2}\langle x, x\rangle_{B} \quad \text { and } \quad{ }_{A}\langle x \cdot b, x \cdot b\rangle \leq\|b\|^{2}{ }_{A}\langle x, x\rangle \text {. }
$$


Morita equivalence of $\mathrm{C}^{*}$-algebras is interesting because such $\mathrm{C}^{*}$-algebras have the same representation theory and the same ideal structure. They need not be the same algebra, however. The following proposition shows that isomorphic $\mathrm{C}^{*}$-algebras are Morita equivalent; we will later see examples of Morita equivalent $\mathrm{C}^{*}$-algebras that are not isomorphic.

Proposition 2.3.3. Isomorphic $C^{*}$-algebras are Morita equivalent.

Proof. Let $A$ and $B$ be two $\mathrm{C}^{*}$-algebras, and suppose $\phi: A \rightarrow B$ is an isomorphism between them. Let $X=B$ be an $A-B$ bimodule equipped with the actions

$$
x \cdot b:=x b \quad a \cdot x:=\phi(a) x
$$

and inner products

$$
\langle x, y\rangle_{B}:=x^{*} y \quad{ }_{A}\langle x, y\rangle:=\phi^{-1}\left(x y^{*}\right)
$$

We will show that these actions and inner products turn $X$ into an equivalence bimodule, and thus $A$ and $B$ are Morita equivalent. Example 2.2.6 shows that $X$ is a full right Hilbert $B$-module. We check the conditions to see that it is a full left Hilbert $A$-module:

(i) ${ }_{A}\langle\lambda x+\mu y, z\rangle=\phi^{-1}\left((\lambda x+\mu y) z^{*}\right)=\lambda \phi^{-1}\left(x z^{*}\right)+\mu \phi^{-1}\left(y z^{*}\right)=\lambda_{A}\langle x, z\rangle+\mu_{A}\langle y, z\rangle$

(ii) ${ }_{A}\langle a \cdot x, y\rangle={ }_{A}\langle\phi(a) x, y\rangle=\phi^{-1}\left(\phi(a) x y^{*}\right)=a \phi^{-1}\left(x y^{*}\right)=a_{A}\langle x, y\rangle$

(iii) ${ }_{A}\langle x, y\rangle^{*}=\phi^{-1}\left(x y^{*}\right)^{*}=\phi^{-1}\left(y x^{*}\right)={ }_{A}\langle y, x\rangle$

(iv) ${ }_{A}\langle x, x\rangle=\phi^{-1}\left(x x^{*}\right)$; since $x x^{*} \geq 0$ as an element of $B, \phi^{-1}\left(x x^{*}\right) \geq 0$.

(v) ${ }_{A}\langle x, x\rangle=0 \Longrightarrow \phi^{-1}\left(x x^{*}\right)=0 \Longrightarrow x x^{*}=0 \Longrightarrow x=0$

Thus $X$ is a inner product $A$-module; its completeness follows from the completeness under the $B$-norm. It is full because products of the form $x y^{*}$ are dense in $B$, and $\phi^{-1}$ is an isomorphism. We verify the last two conditions of Definition 2.3.1 by straightforward calculation:

(ii) $\left\langle a \cdot b, b^{\prime}\right\rangle_{B}=(\phi(a) b)^{*} b^{\prime}=b^{*} \phi\left(a^{*}\right) b^{\prime}=\left\langle b, a^{*} \cdot b^{\prime}\right\rangle_{B}$ and ${ }_{A}\left\langle b \cdot c, b^{\prime}\right\rangle=\phi^{-1}\left(b c\left(b^{\prime}\right)^{*}\right)=\phi^{-1}\left(b\left(b^{\prime} c^{*}\right)^{*}\right)=$ ${ }_{A}\left\langle b, b^{\prime} \cdot c^{*}\right\rangle$ 
(iii) ${ }_{A}\langle a, b\rangle \cdot c=\phi^{-1}\left(a b^{*}\right) \cdot c=\phi\left(\phi^{-1}\left(a b^{*}\right) c=a b^{*} c=a \cdot\langle b, c\rangle_{B}\right.$

Thus isomorphic $\mathrm{C}^{*}$-algebras are also Morita equivalent.

Many Morita equivalences arise from completing so called pre-equivalence structures. We will define such a structure here and then describe its completion.

Definition 2.3.4. Let $A_{0} \subset A$ and $B_{0} \subset B$ be dense *-subalgebras of the $\mathrm{C}^{*}$-algebras $A$ and $B$. An $A_{0}-B_{0}$ pre-equivalence bimodule $X_{0}$ is an $A_{0}-B_{0}$ bimodule satisfying:

(i) $X_{0}$ is a left pre-inner product $A_{0}$-module, and a right pre-inner product $B_{0}$-module,

(ii) $A_{0}\left\langle X_{0}, X_{0}\right\rangle$ spans a dense ideal of $A$, and $\left\langle X_{0}, X_{0}\right\rangle_{B_{0}}$ spans a dense ideal of $B$,

(iii) for all $a \in A_{0}, b \in B_{0}$, and $x \in X_{0}$,

$$
\langle a \cdot x, a \cdot x\rangle_{B} \leq\|a\|^{2}\langle x, x\rangle_{B} \quad \text { and } \quad{ }_{A}\langle x \cdot b, x \cdot b\rangle \leq\|b\|^{2}{ }_{A}\langle x, x\rangle
$$

(iv) for all $x, y, z \in X_{0}$,

$$
{ }_{A}\langle x, y\rangle \cdot z=x \cdot\langle y, z\rangle_{B} .
$$

Remark 2.3.5. By a pre-inner product module, we mean a module $X_{0}$ with an inner product satisfying conditions (i)-(iv) of Definition 2.2.1.

Remark 2.3.6. Notice the similarities with Definition 2.3.1: conditions (i) and (ii) together setup condition (i) for Morita equivalence, condition (iii) is drawn from Equation 2.6, and condition (iv) is the same as Equation 2.5. The only substantial difference is the use of dense $*$-subalgebras and the allowance that the inner products only induce semi-norms.

Proposition 2.3.7. Suppose $X_{0}$ is an $A_{0}-B_{0}$ pre-equivalence bimodule for dense *-subalgebras $A_{0} \subset A$ and $B_{0} \subset B$. Then there exists an $A-B$ equivalence bimodule $X$ and an $A_{0}-B_{0}$ bimodule homomorphism $q: X_{0} \rightarrow X$ such that $q\left(X_{0}\right)$ is dense and, for all $x, y \in X_{0}$,

$$
\langle q(x), q(y)\rangle_{B}=\langle x, y\rangle_{B_{0}} \quad \text { and } \quad{ }_{A}\langle q(x), q(y)\rangle={ }_{A_{0}}\langle x, y\rangle
$$


Proof. Showing that $X$ is a Hilbert module requires the following lemma.

Lemma 2.3.8. If $X_{0}$ is an $A_{0}-B_{0}$ pre-equivalence bimodule, then for all $x \in X_{0}$,

$$
\left\|_{A_{0}}\langle x, x\rangle\right\|=\left\|\langle x, x\rangle_{B_{0}}\right\|
$$

Proof. Choose $x \in X_{0}$. Then

$$
\begin{gathered}
\\
\left\|\langle x, x\rangle_{B_{0}}\right\|^{2}=\left\|\langle x, x\rangle_{B_{0}}\langle x, x\rangle_{B_{0}}\right\|=\left\|\left\langle x, x \cdot\langle x, x\rangle_{B_{0}}\right\rangle_{B_{0}}\right\| \\
=\left\|\left\langle x, A_{0}\langle x, x\rangle \cdot x\right\rangle_{B_{0}}\right\| \leq\left\|\langle x, x\rangle_{B_{0}}\right\|^{\frac{1}{2}}\left\|_{A_{0}}\langle x, x\rangle\right\|\left\|\langle x, x\rangle_{B_{0}}\right\|^{\frac{1}{2}},
\end{gathered}
$$

where the last inequality is due to Equation 2.2. We cancel the factor of $\left\|\langle x, x\rangle_{B_{0}}\right\|$ to get

$$
\left\|\langle x, x\rangle_{B_{0}}\right\| \leq\left\|_{A_{0}}\langle x, x\rangle\right\|
$$

Repeating the argument with the roles of $A_{0}$ and $B_{0}$ swapped proves the lemma.

Lemma 2.3.8 shows that the semi-norms induced by the $A_{0}$ and $B_{0}$ inner products coincide, and hence $X$ is simultaneously a left Hilbert $A$-module and a right Hilbert $B$-module. Condition (ii) of Definition 2.3.4 shows that the Hilbert modules are full. Since conditions (iii) and (iv) hold on $X_{0}$, they also hold in $X$, and thus by applying Proposition 2.3.2, we have satisfied the remaining conditions in Definition 2.3.1. Hence $X$ is an $A-B$ equivalence bimodule as desired.

\subsubsection{Equivalence Relation}

As the terminology suggests, Morita equivalence is in fact an equivalence relation. We will demonstrate the reflexive and symmetric conditions using two basic examples, while transitivity will require a more complicated construction known as the balanced tensor product.

Example 2.3.9. A $\mathrm{C}^{*}$-algebra $A$ is Morita equivalent to itself via the bimodule $A$; the actions are multiplication in $A$, while the inner products are given by

$$
{ }_{A}\langle a, b\rangle=a b^{*} \quad\langle a, b\rangle_{A}=a^{*} b .
$$


Proof. As seen in Example 2.2.6, $A$ is a full right Hilbert $A$-module; a completely similar proof shows that $A$ is also a full left Hilbert $A$-module under the given action and inner product. We verify Equations 2.4 and 2.5 by calculating:

(ii) $\langle a \cdot b, c\rangle_{A}=(a b)^{*} c=b^{*} a^{*} c=\left\langle b, a^{*} \cdot c\right\rangle_{A}$ and ${ }_{A}\langle b \cdot a, c\rangle=b a c^{*}=b\left(a^{*} c\right)^{*}={ }_{A}\left\langle b, a^{*} \cdot c\right\rangle$.

(iii) ${ }_{A}\langle a, b\rangle \cdot c=a b^{*} c=a \cdot\langle b, c\rangle_{A}$

Example 2.3.10. Suppose that $X$ is an $A$-B equivalence bimodule. Consider $\widetilde{X}$, the conjugate vector space of $X$; that is, $\widetilde{X}$ is the same space as $X$, but with an additive bijection $b: X \rightarrow \widetilde{X}$ such that $b(\lambda \cdot x)=\bar{\lambda} \cdot b(x)$.

We can then make $\widetilde{X}$ into a $B$ - $A$ equivalence bimodule via the actions

$$
b \cdot b(x)=b\left(x \cdot b^{*}\right) \quad b(x) \cdot a=b\left(a^{*} \cdot x\right)
$$

and the inner products

$$
{ }_{B}\langle b(x), b(y)\rangle=\langle x, y\rangle_{B} \quad\langle b(x), b(y)\rangle_{A}={ }_{A}\langle x, y\rangle .
$$

Proof. First we show that $\widetilde{X}$ is a right Hilbert $A$-module; the proof for the left Hilbert $B$-module is similar:

(i) $\langle b(x), \lambda b(y)+\mu b(z)\rangle_{A}=\langle b(x), b(\bar{\lambda} \cdot y+\bar{\mu} \cdot z)\rangle_{A}={ }_{A}\langle x, \bar{\lambda} y+\bar{\mu} z\rangle=\lambda_{A}\langle x, y\rangle+\mu_{A}\langle x, z\rangle=$ $\lambda\langle b(x), b(y)\rangle_{A}+\mu\langle b(x), b(z)\rangle_{A}$

(ii) $\langle b(x), b(y) \cdot a\rangle_{A}=\left\langle b(x), b\left(a^{*} \cdot y\right)\right\rangle_{A}={ }_{A}\left\langle x, a^{*} \cdot y\right\rangle={ }_{A}\left\langle a^{*} y, x\right\rangle^{*}=\left(a^{*}{ }_{A}\langle y, x\rangle\right)^{*}={ }_{A}\langle x, y\rangle a=$ $\langle b(x), b(y)\rangle_{A} a$

(iii) $\langle b(x), b(y)\rangle_{A}^{*}={ }_{A}\langle x, y\rangle^{*}={ }_{A}\langle y, x\rangle=\langle b(y), b(x)\rangle_{A}$

(iv) $\langle b(x), b(x)\rangle_{A}={ }_{A}\langle x, x\rangle \geq 0$

$(\mathrm{v})\langle b(x), b(x)\rangle_{A}=0 \Longrightarrow{ }_{A}\langle x, x\rangle=0 \Longrightarrow x=0$ 
Thus $\widetilde{X}$ is an inner product $A$ module; it is complete because the norm induced by the inner product coincides with the norm induced on $X$ by its $A$-valued inner product, and therefore $\widetilde{X}$ is a right Hilbert $A$-module. Fullness follows from the fact that the $A$-valued inner product on $\widetilde{X}$ corresponds with that on $X$, and $X$ is full as a left Hilbert $A$-module.

The second two conditions of Definition 2.3.1 are satisfied as follows:

(ii) $\langle b \cdot b(x), b(y)\rangle_{A}=\left\langle b\left(x \cdot b^{*}\right), b(y)\right\rangle_{A}={ }_{A}\left\langle x \cdot b^{*}, y\right\rangle={ }_{A}\langle x, y \cdot b\rangle=\langle b(x), b(y \cdot b)\rangle_{A}=\left\langle b(x), b^{*} \cdot b(y)\right\rangle_{A}$ and ${ }_{B}\langle b(x) \cdot a, b(y)\rangle={ }_{B}\left\langle b\left(a^{*} \cdot x\right), b(y)\right\rangle=\left\langle a^{*} \cdot x, y\right\rangle_{B}=\langle x, a \cdot y\rangle_{B}={ }_{B}\langle b(x), b(a \cdot y)\rangle={ }_{B}\left\langle b(x), b(y) \cdot a^{*}\right\rangle$

(iii) ${ }_{B}\langle b(x), b(y)\rangle \cdot b(z)=b\left(z \cdot{ }_{B}\langle b(x), b(y)\rangle^{*}\right)=b\left(z \cdot\langle y, x\rangle_{B}\right)=b\left({ }_{A}\langle z, y\rangle \cdot x\right)=b(x) \cdot{ }_{A}\langle z, y\rangle^{*}=$ $b(x) \cdot\langle b(y), b(z)\rangle_{A}$

Thus Morita equivalence is both symmetric and reflexive. We now introduce the balanced tensor product, which will be the key construction for proving the transitivity of Morita equivalence.

Remark 2.3.11. We will use the notation $X \odot Y$ for the algebraic tensor product of $X$ and $Y$.

Definition 2.3.12. Let $X$ be a right Hilbert $B$-module and $Y$ be a left Hilbert $B$-module. The B-balanced tensor product is defined to be

$$
X \odot_{B} Y:=\frac{X \odot Y}{N}
$$

where

$$
N:=\operatorname{span}\{(x \cdot b) \otimes y-x \otimes(b \cdot y): x, y \in X \text { and } b \in B\}
$$

Proposition 2.3.13. Let $X$ be an $A-B$ equivalence bimodule, and $Y$ be a $B-C$ equivalence bimodule. Then $Z=X \odot_{B} Y$ is an $A-C$ pre-equivalence bimodule under the pre-inner products

$$
\begin{aligned}
& \left\langle\left\langle x \otimes_{B} y, z \otimes_{B} w\right\rangle_{C}=\left\langle\langle z, x\rangle_{B} \cdot y, w\right\rangle_{C}\right. \\
& { }_{A}\left\langle\left\langle x \otimes_{B} y, z \otimes_{B} w\right\rangle=_{A}\left\langle x, z \cdot{ }_{B}\langle w, y\rangle\right\rangle .\right.
\end{aligned}
$$


Remark 2.3.14. The inner products given do turn $Z$ into a left pre-inner product $A$-module and a right pre-inner product $C$-module, but the proof of this fact is surprisingly deep. We refer the reader to Proposition 3.16 of [15] for a full proof, and then freely assume this in our proof below.

Proof. We must verify the four conditions of Definition 2.3.4; the first condition is assumed. For condition (ii) we consider the span of $\left\langle\langle Z, Z\rangle_{C}\right.$. This is equivalent to $\left\langle\langle X, X\rangle_{B} \cdot Y, Y\right\rangle_{C}$, but since $X$ is a full right Hilbert $B$-module, $\langle X, X\rangle_{B}$ is dense in $B$, and thus $\langle X, X\rangle_{B} \cdot Y$ is dense in $Y$, and thus $\left\langle\langle Z, Z\rangle_{C}\right.$ is dense in $C$. Similarly, ${ }_{A}\langle\langle Z, Z\rangle\rangle$ is dense in $A$.

For condition (iv), we calculate:

$$
\left(x \otimes_{B} y\right) \cdot\left\langle\left\langle x_{1} \otimes_{B} y_{1}, x_{2} \otimes_{B} y_{2}\right\rangle_{C}=x \otimes_{B}\left(y \cdot\left\langle\left\langle x_{2}, x_{1}\right\rangle_{B} \cdot y_{1}, y_{2}\right\rangle_{C}\right)=x \otimes_{B}\left({ }_{B}\left\langle y,\left\langle x_{2}, x_{1}\right\rangle_{B} \cdot y_{1}\right\rangle \cdot y_{2}\right),\right.
$$

where the second equality uses the third condition of the Morita equivalence between $B$ and $C$,

$$
=\left(x \cdot{ }_{B}\left\langle y,\left\langle x_{2}, x_{1}\right\rangle_{B} \cdot y_{1}\right\rangle\right) \otimes_{B} y_{2}
$$

where we use the equivalence from the subspace $N$ in the balanced tensor product,

$$
=\left(x \cdot{ }_{B}\left\langle y, y_{1}\right\rangle\left\langle x_{1}, x_{2}\right\rangle_{B}\right) \otimes_{B} y_{2}=\left(x \cdot\left\langle x_{1} \cdot{ }_{B}\left\langle y_{1}, y\right\rangle, x_{2}\right\rangle_{B}\right) \otimes_{B} y_{2}=\left({ }_{A}\left\langle x, x_{1} \cdot{ }_{B}\left\langle y_{1}, y\right\rangle\right\rangle \cdot x_{2}\right) \otimes_{B} y_{2}
$$

where the last equality uses the third condition of the Morita equivalence between $A$ and $B$,

$$
={ }_{A}\left\langle\left\langle x \otimes_{B} y, x_{1} \otimes_{B} y_{1}\right\rangle\right\rangle \cdot\left(x_{2} \otimes_{B} y_{2}\right) .
$$

Recall now that Proposition 2.3.2 draws an equivalence between condition (ii) of Definition 2.3.1 and condition (iii) of Definition 2.3.4. This equivalence of conditions extends to pre-equivalence bimodules if the $A_{0}$ and $B_{0}$ are $\mathrm{C}^{*}$-algebras in their own right; thus for our current case, we will check the former condition for both pre-inner products:

$$
\begin{gathered}
\left\langle\left\langle a \cdot x \otimes_{B} y, x_{1} \otimes_{B} y_{1}\right\rangle_{C}=\left\langle\left\langle x_{1}, a \cdot x\right\rangle_{B} \cdot y, y_{1}\right\rangle_{C}=\left\langle\left\langle a^{*} \cdot x_{1}, x\right\rangle_{B} \cdot y, y_{1}\right\rangle_{C}=\left\langle\left\langle x \otimes_{B} y, a^{*} \cdot x_{1} \otimes_{B} y_{1}\right\rangle_{C}\right.\right. \\
{ }_{A}\left\langle\left\langle x \otimes_{B} y \cdot c, x_{1} \otimes_{B} y_{1}\right\rangle\right\rangle={ }_{A}\left\langle x, x_{1} \cdot{ }_{B}\left\langle y_{1}, y \cdot c\right\rangle\right\rangle={ }_{A}\left\langle x, x_{1} \cdot{ }_{B}\left\langle y_{1} \cdot c^{*}, y\right\rangle\right\rangle={ }_{A}\left\langle\left\langle x \otimes_{B} y, x_{1} \otimes_{B} y_{1} \cdot c^{*}\right\rangle\right\rangle
\end{gathered}
$$

Thus we have verified all the conditions of Definition 2.3.4, and therefore $Z$ is an $A-C$ pre-equivalence bimodule. 
Corollary 2.3.15. The completion (as in Proposition 2.3.7) of the balanced tensor product $Z=$ $X \odot_{B} Y$ forms an equivalence bimodule between $A$ and $C$. We call this bimodule the internal tensor product, and denote it as $X \otimes_{B} Y$.

We have thus proved the following proposition:

Proposition 2.3.16. Morita equivalence of $C^{\star}$-algebras is an equivalence relation.

The internal tensor product will play a big role in Chapter 5; for now, we will shift our focus to examples of Morita equivalences involving irrational rotation algebras.

\subsubsection{Morita equivalences of $A_{\alpha}$}

In this section we will highlight some Morita equivalences involving the irrational rotation algebras. These examples will help motivate and construct our projective multiresolution analyses later on.

We know when two irrational rotation algebras are isomorphic by Proposition 2.1.3. Since Morita equivalence is a weaker condition, it is natural to wonder if there are further values of $\beta$ for which $A_{\alpha}$ and $A_{\beta}$ are Morita equivalent. This question was also answered by Rieffel in [17], and we state his result here.

Proposition 2.3.17. [17] Let $\alpha$ and $\beta$ be irrational numbers. The following are equivalent:

(1) $A_{\alpha}$ is Morita equivalent to $A_{\beta}$

(2) $\alpha$ and $\beta$ are in the same orbit of the action of $G L(2, \mathbb{Z})$ on irrational numbers via fractional linear transformations.

The action described above is as follows: Let $g=\left(\begin{array}{cc}a & b \\ c & d\end{array}\right)$, and define $\phi_{g}(\alpha)=\frac{a \alpha+b}{c \alpha+d}$. For example, if $g=\left(\begin{array}{cc}0 & 1 \\ 1 & 0\end{array}\right)$, then $\alpha \mapsto \frac{1}{\alpha}$, and thus the theorem states that $A_{\alpha}$ is Morita equivalent to $A_{\frac{1}{\alpha}}$ 
In the general case, this result is difficult to prove. For individual values of $\alpha$ and $\beta$, however, we can see the Morita equivalence explicitly for the correct bimodule and inner products, as in the following example:

Example 2.3.18. [17] Let $X=C_{c}(\mathbb{R})$, and define $C=C_{c}(\mathbb{Z} \alpha, \mathbb{R} / \mathbb{Z})$ and $D=C_{c}(\mathbb{Z}, \mathbb{R} / \mathbb{Z} \alpha)$. Then $X$ can be made into a pre-equivalence bimodule for $C$ and $D$ with the actions of pointwise multiplication by functions of period 1 and $\alpha$, and the inner products

$$
{ }_{C}\langle f, g\rangle(m, r)=\sum_{i=1}^{n} f(r-n) \bar{g}(r-n-m \alpha) \quad\langle f, g\rangle_{D}(m, r)=\sum_{i=1}^{n} \bar{f}(r-n \alpha) g(r-n \alpha-m) .
$$

When completed as in Proposition 2.3.7, $X$ is an equivalence bimdoule implementing the Morita equivalence between $A_{\alpha}$ and $A_{\frac{1}{\alpha}}$.

The following example of a Morita equivalence is a more general case. It is also due to Rieffel, and will be the motivation behind our first example of a projective multiresolution analysis.

Example 2.3.19. [16] Let $Y$ be a compact space, let $T: Y \rightarrow Y$ be a homeomorphism, and suppose $M$ is the mapping torus for $Y$, that is, $M=Y \times \mathbb{R} / \sim$, where the equivalence is given by $(y, r) \sim(T y, r+1)$. Then $C_{c}(Y \times \mathbb{R})$ can be given a left Hilbert $C_{c}(Y \times \mathbb{Z})$-module structure and a right Hilbert $C_{c}(M \times \mathbb{R})$-module structure via the actions:

$$
\begin{gathered}
f \cdot \Psi(y, r)=\sum_{j \in \mathbb{Z}} f(y, j) \Psi\left(T^{j} y, r-j\right) \\
\Psi \cdot g(y, r)=\int_{\mathbb{R}} \Psi(y, r+t) g\left(\phi_{1}(y, r+t),-t\right) d t,
\end{gathered}
$$

where $\phi_{1}: Y \times \mathbb{R} \rightarrow M$, as well as the inner products:

$$
\begin{gathered}
C_{c}(Y \times \mathbb{Z})\left\langle\Psi_{1}, \Psi_{2}\right\rangle(y, n)=\int_{\mathbb{R}} \Psi_{1}(y, t) \overline{\Psi_{2}\left(T^{n} y, t-n\right)} d t \\
\left\langle\Psi_{1}, \Psi_{2}\right\rangle_{C_{c}(M \times \mathbb{R})}\left(\phi_{1}(y, r), t\right)=\sum_{j \in \mathbb{Z}} \overline{\Psi_{1}\left(T^{j} y, r-j\right)} \Psi_{2}\left(T^{j} y, r-j+t\right)
\end{gathered}
$$

Under these actions and inner products, $C_{c}(Y \times \mathbb{Z})$ and $C_{c}(M \times \mathbb{R})$ have a pre-equivalence structure; when completed as in Proposition 2.3.7, the $\mathrm{C}^{*}$-algebras $C(Y) \times \mathbb{Z}$ and $C(M) \times \mathbb{R}$ are then Morita equivalent to each other. 
Remark 2.3.20. We will soon consider the specific case where $Y=\mathbb{T}$, and $T: \mathbb{T} \rightarrow \mathbb{T}$ is defined by

$$
T(z)=e^{2 \pi i \alpha} z
$$

For this $Y$ and $T, C_{c}(Y \times \mathbb{Z})$ completes to the irrational rotation algebra $A_{\alpha}$. 


\section{Chapter 3}

\section{Projective Multiresolution Analyses}

\subsection{Defining Projective Multiresolution Analyses over $A_{\alpha}$}

\subsubsection{PMRAs over $C\left(\mathbb{T}^{2}\right)$}

In their 2004 paper, [13], Packer and Rieffel defined projective multiresolution analyses in the context of the algebra $\mathcal{A}=C\left(\mathbb{T}^{n}\right)$. We examine their definition and highlight some properties of such projective multiresolution analyses before extending the idea to $A_{\alpha}$-modules.

Packer and Rieffel worked with the following space for their projective multiresolution analyses.

Definition 3.1.1. [13] We let $\Xi$ denote the set of bounded continuous functions on $\mathbb{R}^{n}$ for which there is a constant, $K$, such that $\sum_{p \in \mathbb{Z}^{n}}|\xi(x-p)|^{2} \leq K$ for each $x \in \mathbb{R}^{n}$, and furthermore such that the function defined by this sum is continuous. The norm is given by

$$
\|\xi\|_{\mathcal{A}}=\left\|\langle\xi, \xi\rangle_{\mathcal{A}}\right\|^{1 / 2}=\sup _{x}\left(\sum_{p \in \mathbb{Z}^{n}}|\xi(x-p)|^{2}\right)^{1 / 2} .
$$

Note that $\Xi \subset L^{2}\left(\mathbb{R}^{2}\right)$. In the following definition, let $A$ be an $n \times n$ dilation matrix, in other words a matrix, all of whose eigenvalues have modulus greater than one, and set

$$
(D \xi)(x)=|\operatorname{det}(A)|^{-1 / 2} \xi\left(\left(A^{t}\right)^{-1} x\right),
$$

where $A^{t}$ is the transpose of $A$.

Definition 3.1.2. [13] A projective multiresoultion analysis for dilation by $A$ is a family $\left\{V_{j}\right\}_{j \in \mathbb{Z}}$ of subspaces of $\Xi$ such that 
(1) $V_{0}$ is a projective $\mathcal{A}$-submodule of $\Xi$,

(2) $V_{j}=D^{j}\left(V_{0}\right)$ for all $j$,

(3) $V_{j} \supset V_{j-1}$ for all $j$,

(4) $\cup^{\infty} V_{j}$ is dense in $\Xi$,

(5) $\cap_{-\infty}^{\infty} V_{j}=\{0\}$

Remark 3.1.3. Packer and Rieffel showed in their context that condition (5) was redundant, given conditions (1) and (2). In addition, given the existence of a $\xi \in V_{0}$ such that $\xi(0) \neq 0$, they showed that conditions (1), (2), and (3) imply condition (4). For details, see [13].

The essence of [13] was to create examples of projective multiresolution analyses whose initial modules were not free. Our goal will be to create a simple example of a PMRA using $A_{\alpha}$-modules. However, we will have to modify the definition of projective multiresolution analysis given in [13] in order to account for our highly noncommutative setting. For our example, we will rely upon a densely defined dilation operator to construct the subspaces $\left\{V_{i}\right\}$, but the dilation itself will not be part of the definition.

\subsubsection{Projective Multiresolution Analyses over $A_{\alpha}$}

In order to form projective multiresolution analyses for modules over noncommutative $\mathrm{C}^{*}$ algebras, it will be convenient to relax some of the restrictions, namely we will not insist that the function $D$ be a dilation in the strictest sense; rather, we only need a map $D: \Xi \rightarrow \Xi$ satsifying certain conditions, to be determined later. Indeed our operator $D$ will be densely defined and used as a tool in the construction of our projective multiresolution analyses.

Definition 3.1.4. Let $\Xi$ be a countably-generated left $A_{\alpha}$-module. A projective multiresolution analysis (PMRA) for $\Xi$ over $A_{\alpha}$ is a family $\left\{V_{j}\right\}_{j \in \mathbb{Z}}$ of closed subspaces of $\Xi$ such that

(i) $V_{j}$ is a projective $A_{\alpha}$-submodule of $\Xi, \forall j \geq 0$ 
(ii) $V_{j} \supset V_{j-1}$ for all $j$,

(iii) $\cup_{j}^{\infty} V_{j}$ is dense in $\Xi$,

(iv) $\cap_{-\infty}^{\infty} V_{j}=\{0\}$.

In order to work with more general $A_{\alpha}$-modules, we will want to work with function spaces over direct products, for example $C_{c}(Y \times \mathbb{R})$, as seen in Example 2.3.19. Hence we want to allow for operators that dilate some coordinates of the direct product, but not others. This is how we will relax our requirements on $D$. In fact, the overall strategy will be to work with products $Y \times \mathbb{R}$ and do all our work in the real variable, while ignoring the space $Y$ as much as is possible.

Our goal now will be to form an example of such a projective multiresolution analysis. The simplest such example will begin with an initial module, $V_{0}$, that is free.

\subsection{A PMRA over $A_{\alpha}$ that is initially free}

Our PMRA will require three ingredients: the $A_{\alpha}$-module $\Xi$ for which we are constructing the PMRA, the densely defined dilation-like operator $D$ which will aid in the construction of our PMRA, and a so-called scaling function that we will use to construct the initial free $A_{\alpha}$-module. We begin with $\Xi$.

\subsubsection{The Morita Equivalence}

Let us recall Example 2.3.19, which created a pre-equivalence structure between the spaces $C_{c}(Y \times \mathbb{Z})$ and $C_{c}(M \times \mathbb{R})$. We will now set $Y=\mathbb{T}$, and define $T: \mathbb{T} \rightarrow \mathbb{T}$ by $T(z)=e^{2 \pi i \alpha} z$.

It is known that $C_{c}(\mathbb{T} \times \mathbb{Z})$ can be viewed as a dense ${ }^{*}$-subalgebra of an irrational rotation algebra $A_{\alpha}$. If we complete the bimodule structure under the norm given by the $C_{c}(\mathbb{T} \times \mathbb{Z})$-valued inner product,

$$
\left\langle\Psi_{1}, \Psi_{2}\right\rangle_{C_{c}(\mathbb{T} \times \mathbb{Z})}(t, n)=\int_{\mathbb{R}} \Psi_{1}(t, r) \overline{\Psi_{2}\left(e^{2 \pi i \alpha n} t, r-n\right)} d r
$$

we obtain the Morita equivalence

$$
A_{\alpha}-\Xi-C(M) \times \mathbb{R}
$$


where $\Xi$ is the completion of $C_{c}(\mathbb{T} \times \mathbb{R})$ under the $A_{\alpha}$ norm. Thus we can view $\Xi$ as a full left Hilbert $A_{\alpha}$-module, under the above inner product and the action

$$
f \cdot \Psi(t, r)=\sum_{j \in \mathbb{Z}} f(t, j) \Psi\left(e^{2 \pi i \alpha j} t, r-j\right)
$$

for $f \in A_{\alpha}, \Psi \in \Xi$. It is this $\Xi$ on which we will build our projective multiresolution analysis. Since it is known that $C(M) \times \mathbb{R}$ is stably isomorphic to $A_{\alpha}$, one expects $\Xi$ to be a countably generated free module.

\subsubsection{The Dilation}

We will now consider a dilation-like operator defined on a dense subset of $\Xi$, which will allow us to construct the closed subspaces $\left\{V_{i}\right\}$ that form our projective multiresolution analysis.

Definition 3.2.1. Define the operator $D: C_{c}(\mathbb{T} \times \mathbb{R}) \rightarrow C_{c}(\mathbb{T} \times \mathbb{R})$ to be the operator that dilates the second argument by 2 ; that is,

$$
D \Psi(t, r)=\sqrt{2} \Psi(t, 2 r)
$$

for all $\Psi \in C_{c}(\mathbb{T} \times \mathbb{R})$.

Our plan for constructing the PMRA will be to take a dense subspace of the initial module $V_{0}$ and apply powers of $D$ to it, then taking the closure to form the subspaces $V_{j}$ for all $j \in \mathbb{Z}$. Thus it remains only to determine the initial module $V_{0}$.

\subsubsection{The Scaling Function}

Our idea for the construction of the initial free $A_{\alpha}$-submodule of $\Xi$ is to find a particular function $\phi \in \Xi$, and then to look at the action of $A_{\alpha}$ on that single function. This will of course require $\phi$ to have specific properties, especially to obtain a projective multiresolution analysis from such an initial module.

First, in order for this $\phi$ to be a generator for the free module $V_{0}$, we require that

$$
\langle\phi, \phi\rangle_{A_{\alpha}}=i d_{A_{\alpha}}
$$


On the left side we apply the formula for the inner product from the original Morita equivalence to obtain

$$
\int_{\mathbb{R}} \phi(t, r) \overline{\phi\left(e^{2 \pi i \alpha n} t, r-n\right)} d r=\delta(0, n)
$$

where $\delta(0, n)$ is the Kronecker delta. We can simplify the situation even further by choosing $\phi$ to be independent of the circle variable $t$, thus reducing our condition to

$$
\int_{\mathbb{R}} \phi(r) \overline{\phi(r-n)} d r=\delta(0, n)
$$

In addition, in order for us to obtain a projective multiresolution structure from this setting, we will have to satisfy condition (ii) of Definition 3.1.4. In particular, we will have to have that

$$
\phi \in V_{0} \subset V_{1}
$$

Let us suppose that we have selected such a $\phi$. Then by employing the action of $A_{\alpha}$ on $\Xi$ obtained from the bimodule structure, we can write

$$
V_{0}=A_{\alpha} \cdot \phi=\overline{\left\{\sum_{k \in \mathbb{Z}} f(t, k) \phi(r-k): f \in C_{c}(\mathbb{T} \times \mathbb{Z})\right\}}
$$

Along the same lines, we can describe the module $V_{1}$ in greater detail as well.

$$
V_{1}=\overline{\left\{\sum_{k \in \mathbb{Z}} \sqrt{2} f(t, k) \phi(2 r-k): f \in C_{c}(\mathbb{T} \times \mathbb{Z})\right\}}
$$

And similarly, for any $j \in \mathbb{Z}$, we have

$$
V_{j}=\overline{\left\{\sum_{k \in \mathbb{Z}}(\sqrt{2})^{j} f(t, k) \phi\left(2^{j} r-k\right): f \in C_{c}(\mathbb{T} \times \mathbb{Z})\right\}}
$$

But now since we want $V_{0} \subset V_{1}$ and $\phi \in V_{0}$, then we should have $\phi \in V_{1}$. This will happen if $\phi$ satisfies the scaling function equation

$$
\phi(r)=\sum_{j \in \mathbb{Z}} c_{j} \phi(2 r-j)
$$

for finitely many nonzero constants $c_{j}$. There are several functions that satisfy both this and Equation 3.1; the following is a classic example. 
Example 3.2.2. [2] Suppose we set all constants $c_{j}=0$ in the scaling function equation except for

$$
c_{0}=\frac{1+\sqrt{3}}{4}, c_{1}=\frac{3+\sqrt{3}}{4}, c_{2}=\frac{3-\sqrt{3}}{4}, c_{3}=\frac{1-\sqrt{3}}{4}
$$

Then there is a well-known function of norm one, $\phi$, that satisfies equation 3.2 called the $D 4$ scaling function, due to Daubechies. It satisfies all of the properties we seek in our scaling function. Indeed, Daubechies has constructed an infinite family of such compactly supported scaling functions in [2]; we give as example here the easiest one to describe.

\subsubsection{The PMRA}

We briefly summarize the elements for our projective multiresolution analysis over the irrational rotation algebra $A_{\alpha}$. We have chosen our $A_{\alpha}$-module to be $\Xi$, the completion of $C_{c}(\mathbb{T} \times \mathbb{R})$ in the norm induced by the $A_{\alpha}$-valued inner product. Our densely defined dilation like operator $D$ satisfies

$$
D \psi(t, r)=\sqrt{2} \psi(t, 2 r)
$$

for all $\psi \in C_{c}(\mathbb{T} \times \mathbb{R}) \subset \Xi$. And finally we have chosen a scaling function $\phi \in \Xi$ that satisfies

(1) $\int_{\mathbb{R}} \phi(r) \overline{\phi(r-n)} d r=\delta(0, n)$

(2) $\phi(r)=\sum_{j \in \mathbb{Z}} c_{j} \phi(2 r-j)$ for finitely many nonzero constants $c_{j}$

with Example 3.2.2 in mind as such a scaling function. We then set $V_{0}=A_{\alpha} \cdot \phi$, the action of the irrational rotation algebra on the scaling function alone. Thus $V_{0}$ is a free $A_{\alpha}$-submodule of $\Xi$ that is singly generated by $\phi$.

The family of subspaces is then generated by applying powers of $D$ to a dense subspace of the initial module, namely $V_{j}=\overline{D^{j}\left(C_{c}(\mathbb{T} \times \mathbb{R}) \cdot \phi\right)}$ for all $j \in \mathbb{Z}$. We obtain the following theorem.

Theorem 3.2.3. The family $\left\{V_{j}\right\}_{j \in \mathbb{Z}}$ of subspaces of $\Xi$ forms a projective multiresolution analysis for the $A_{\alpha}$-module $\Xi$.

Proof. We must verify the four conditions in Definition 3.1.4. Several of these are straightforward. 
(i) $V_{0}$ is a projective $A_{\alpha^{-}}$submodule of $\Xi$ : By construction, $V_{0}$ is a singly-generated free $A_{\alpha^{-}}$ submodule of $\Xi$.

(ii) $V_{j-1} \subset V_{j}$ for all $j \in \mathbb{Z}$ : The scaling function $\phi$ was chosen so that $\phi \in V_{1}$, which implies that $V_{0} \subset V_{1}$. Applying powers of the densely defined $D$ to the dense subspaces defining the $V_{j}$ and condition (2) to that relation shows that $V_{j-1} \subset V_{j}$ for all $j \in \mathbb{Z}$.

(iii) $\cup_{j}^{\infty} V_{j}$ is dense in $\Xi$ : To prove the density condition, it will be enough to show that $C_{c}(\mathbb{T} \times \mathbb{R})$ is contained in the closure of this union. We consider the following lemma.

Lemma 3.2.4. Viewed as a subspace of $C_{c}(\mathbb{T} \times \mathbb{R}), C_{c}(\mathbb{R}) \subset \overline{\cup_{j=0}^{\infty} V_{j}}$.

Proof. For all $j \in \mathbb{Z}$, define the space $\mathcal{V}_{j}=V_{j} \cap C_{c}(\mathbb{R})$. Then $D^{j}$ is defined on $\mathcal{V}_{0}$, and

$$
D^{j}\left(\mathcal{V}_{0}\right)=D^{j}\left(V_{0} \cap C_{c}(\mathbb{R})\right)=V_{j} \cap C_{c}(\mathbb{R})=\mathcal{V}_{j}
$$

so that the $\left\{\mathcal{V}_{j}\right\}$ also form a nested family of subspaces. The majority of the results in [13] apply to this family of subspaces, and we can use them to show that

$$
C_{c}(\mathbb{R}) \subset \overline{\bigcup_{j=0}^{\infty} \mathcal{V}_{j}}
$$

But then $\mathcal{V}_{j} \subset V_{j}$ for every $j \in \mathbb{Z}$, and therefore we get

$$
C_{c}(\mathbb{R}) \subset \overline{\bigcup_{j=0}^{\infty} V_{j}}
$$

as desired.

Now, given any $g \in C(\mathbb{T})$, define $M_{g} \in C_{c}(\mathbb{T} \times \mathbb{Z})$ to be given by

$$
M_{g}(t, n)= \begin{cases}g(t) & n=0 \\ 0 & n \neq 0\end{cases}
$$


Then given any $\gamma \in C_{c}(\mathbb{R}) \subset C_{c}(\mathbb{T} \times \mathbb{R})$, applying the action from the Hilbert $A_{\alpha}$-module structure gives us

$$
M_{g} \cdot \gamma(t, r)=\sum_{j \in \mathbb{Z}} M_{g}(t, j) \gamma\left(e^{2 \pi i \alpha j} t, r-j\right)=g(t) \gamma(r)
$$

This combined with Lemma 3.2.4 implies that

$$
g(t) \gamma(r) \in \overline{\bigcup_{j=0}^{\infty} V_{j}}
$$

We can extend this to any finite sum of such products, so that

$$
\sum_{i=1}^{n} g_{i}(t) \gamma_{i}(r) \in \overline{\bigcup_{j=0}^{\infty} V_{j}}
$$

and since sums of these form can be used to approximate all elements of $C_{c}(\mathbb{T} \times \mathbb{R})$ in the uniform norm, we get that

$$
C_{c}(\mathbb{T} \times \mathbb{R}) \subset \overline{\bigcup_{j=0}^{\infty} V_{j}}
$$

as desired.

(iv) $\cap_{-\infty}^{\infty} V_{j}=\{0\}$ : We turn to the following lemma, following [13]:

Lemma 3.2.5. Let $V_{0}=A_{\alpha} \cdot \phi$ be an $A_{\alpha}$-submodule of $\Xi$, and set $V_{j}=\overline{D^{j}\left(C_{c}(\mathbb{T} \times \mathbb{R}) \cdot \phi\right)}$ for all $j$. Then

$$
\bigcap_{-\infty}^{\infty} V_{j}=\{0\}
$$

Proof. Let $\xi \in \cap_{-\infty}^{\infty} V_{j}$. Then in particular, $\xi \in V_{0}=A_{\alpha} \cdot \phi$, so we can write $\xi=f_{\xi} \cdot \phi$ for some $f_{\xi}$ in $A_{\alpha}$. Therefore $f_{\xi} \cdot \phi \in V_{j}$ for all $j \in \mathbb{Z}$.

Now consider for a fixed $g \in C(\mathbb{T})$ the multiplication operator $M_{g}$ defined above. A simple calculation shows that $M_{g}$ commutes with $D$; for any $\Psi \in C_{c}(\mathbb{T} \times \mathbb{R})$ :

$$
\begin{aligned}
& D\left(M_{g} \cdot \Psi(t, r)\right)=D(g(t) \Psi(t, r))=\sqrt{2} g(t) \Psi(t, 2 r) \\
& =g(t)(\sqrt{2} \Psi(t, 2 r))=g(t) D \Psi(t, r)=M_{g} \cdot D \Psi(t, r)
\end{aligned}
$$


We now wish to show that $V_{j}$ is closed under action by $M_{g}$. Since $M_{g}$ and $D$ commute, we have

$$
M_{g} V_{j}=M_{g} \overline{D^{j}\left(C_{c}(\mathbb{T} \times \mathbb{R}) \cdot \phi\right)}=\overline{D^{j}\left(M_{g} C_{c}(\mathbb{T} \times \mathbb{R}) \cdot \phi\right)}
$$

and since $M_{g} C_{c}(\mathbb{T} \times \mathbb{R})=C_{c}(\mathbb{T} \times \mathbb{R})$, we have that $M_{g} V_{j}=V_{j}$. Thus since $f_{\xi} \cdot \phi \in V_{j}$ for all $j \in \mathbb{Z}$, we have for any $g \in C(\mathbb{T})$ that

$$
M_{g} f_{\xi} \cdot \phi \in M_{g} V_{j}=V_{j}
$$

Now, if we view the irrational rotation algebra $A_{\alpha}$ as the crossed product $C(\mathbb{T}) \times_{\alpha} \mathbb{Z}$, then the space $\mathbb{C} \times_{\alpha} \mathbb{Z}$ is a subalgebra of $A_{\alpha}$, consisting of functions of the form

$$
\sum_{n \in \mathbb{Z}} c_{n} U^{n}
$$

for complex-valued constants $c_{n}$. We will consider the conditional expectation $E: C(\mathbb{T}) \times_{\alpha}$ $\mathbb{Z} \rightarrow \mathbb{C} \times_{\alpha} \mathbb{Z}$ given by

$$
E\left(\sum_{n \in \mathbb{Z}} f_{n}(t) U^{n}\right)=\sum_{n \in \mathbb{Z}}\left(\int_{0}^{1} f_{n}(t) d t\right) U^{n}
$$

in other words, the constant coefficients are obtained by integrating the functions $f_{n}(t) \epsilon$ $C(\mathbb{T})$ over the circle.

Since $\mathbb{C} \times_{\alpha} \mathbb{Z}$ is a subalgebra of $A_{\alpha}$ and $V_{0}=A_{\alpha} \cdot \phi$, for any $f \in A_{\alpha}$ we have that

$$
E(f) \cdot \phi \in V_{0}
$$

Recall that for all $j \in \mathbb{Z}$,

$$
V_{j}=\overline{\left\{\sum_{k \in \mathbb{Z}}(\sqrt{2})^{j} f(y, k) \phi\left(2^{j} r-k\right): f \in C_{c}(Y \times \mathbb{Z})\right\}}
$$

Since $\phi$ can be expressed as a finite linear combination of functions of the form

$$
\left\{(\sqrt{2})^{j} \phi\left(2^{j} t-k\right): k \in \mathbb{Z}\right\}
$$

for any $f \in C_{c}(Y \times \mathbb{Z})$, we have for any $j$,

$$
E(f) \cdot \phi \in V_{j}
$$


Therefore by a density argument and the continuity of $E$, we get $E\left(f_{\xi}\right) \cdot \phi \in V_{j}$ for all $j \in \mathbb{Z}$. If we let $f_{\xi}=\sum_{n \in \mathbb{Z}} \xi_{n}(t) U^{n}$, then this implies that

$$
\int_{0}^{1} \xi_{n}(t) d t=0 \text { for all } n \in \mathbb{Z}
$$

But if we now recall 3.3 , the same reasoning applied to $M_{g} f_{\xi}$ shows that for any $g \in C(\mathbb{T})$,

$$
\int_{0}^{1} g(t) \xi_{n}(t) d t=0 \text { for all } n \in \mathbb{Z}
$$

which is enough to show that $\xi_{n}(t)=0$ for all $n \in \mathbb{Z}$. Hence $f_{\xi}$ is identically 0 , and $\xi=f_{\xi} \cdot \phi=0$ as well, and the intersection of the $V_{j}$ is trivial.

Thus all four conditions in Definition 3.1.4 are satsified, and the family $\left\{V_{j}\right\}$ forms a projective multiresolution analysis for $\Xi$ over $A_{\alpha}$.

Thus we have constructed an example of a projective multiresolution analysis for an $A_{\alpha^{-}}$ module in which the initial module is a free $A_{\alpha}$-submodule. One of the main uses of PMRAs is to construct module frames for the module in question; we now turn towards this task.

\subsection{Building The Module Frame}

In this section we use our newly constructed projective multiresolution analysis to construct a module basis for $\Xi$, and notice an isomorphism between this and a well-known Hilbert $A_{\alpha}$-module.

\subsubsection{The Frame}

To construct a module frame for $\Xi$, we will first cite the definition given in [13], adapted from $[5]$. 
Definition 3.3.1. Let $\Xi$ be a right Hilbert $C^{*}$-module over a unital $C *$-algebra $\mathcal{A}$. We say that a countable subset $\left\{\phi_{j}\right\}_{j \in I}$ of $\Xi$ is a standard module frame for $\Xi$ if for every $\xi \in \Xi$,

$$
\langle\xi, \xi\rangle_{\mathcal{A}}=\sum_{j \in I}\left\langle\xi, \phi_{j}\right\rangle_{\mathcal{A}}\left\langle\phi_{j}, \xi\right\rangle_{\mathcal{A}}
$$

where the sum on the right-hand side converges in norm in $\mathcal{A}$.

We will construct our module frame by finding finite sets of functions that span $V_{0}$ and the orthogonal complements $W_{j}$, defined below. Our method will be to construct a wavelet $\psi \in V_{1} \ominus V_{0}$, and then use translations and dilations of that wavelet to construct the frame.

Definition 3.3.2. For any function $f \in \Xi$, define the translation operator $T$ to be

$$
T f(t, r)=f(t, r-1)
$$

Remark 3.3.3. Notice that our translation operator behaves very similarly to our densely defined dilation operator $D$ in that it ignores the circle variable in favor of only acting on the real variable.

With that remark in mind we notice the following relationship between translation $T$ and dilation $D$.

Lemma 3.3.4. With $T$ defined as in Definition 3.3.2 and D defined as in Definition 3.2.1, we have the commutation relation

$$
T \circ D=D \circ T^{2}
$$

Proof. Given any $f \in \Xi$, we have

$$
\begin{gathered}
(T \circ D) f(t, r)=\sqrt{2} T f(t, 2 r)=\sqrt{2} f(t, 2(r-1)) \\
=\sqrt{2} f(t, 2 r-2)=\sqrt{2}\left(T^{2}\right) f(t, 2 r)=\left(D \circ T^{2}\right) f(t, r)
\end{gathered}
$$

We will now consider the orthogonal complements of our nested family of subspaces $\left\{V_{i}\right\}$. 
Definition 3.3.5. For each $i$, define the orthogonal complement of $V_{i}$ in $V_{i+1}$ to be

$$
W_{i}=V_{i+1} \ominus V_{i}
$$

In particular, the space $W_{0}$ is called the wavelet module.

Remark 3.3.6. To build our module frame we will want $V_{i} \oplus W_{i}=V_{i+1}$. However, for Hilbert $\mathrm{C}^{*}$-modules, this is not always the case for orthogonal complements. There are conditions which allow for this to hold, in particular when the modules involved are finitely generated. For our projective multiresolution analysis, starting with a singly-generated $V_{0}$ ensures that the dilated $V_{j}$ are finitely generated, so that our $W_{i}$ behave as we desire.

Now recall that our scaling function $\phi \in V_{0}$ in fact generated $V_{0}$, and in addition, had to satisfy the relation

$$
\phi(r)=\sum_{j \in \mathbb{Z}} c_{j} \phi(2 r-j)
$$

for finitely many nonzero constants $c_{j}$.

Given such a $\phi$, there is a canonical way, due to I. Daubechies in [2], to construct a wavelet $\psi \in W_{0}$ satisfying

$$
\psi(r)=\sum_{j \in \mathbb{Z}} b_{j} \psi(2 r-j)
$$

for finitely many nonzero constants $b_{j}$. In addition, $\psi$ generates the wavelet module $W_{0}$, so that taken together, $\phi$ and $\psi$ generate $V_{1}=V_{0} \oplus W_{0}$.

We now follow classical frame construction and apply combinations of our dilation $D$ and translation $T$ to the wavelet $\psi$ to obtain functions in the remaining $W_{j}$.

Definition 3.3.7. For $j \in \mathbb{Z}_{\geq 0}$, define the function

$$
\psi_{j, k}=D^{j} T^{k} \psi
$$

for all $k \in \mathbb{Z}_{\geq 0}$.

Lemma 3.3.8. For every $j \in \mathbb{Z}_{\geq 0}, \psi_{j, k} \in W_{j}$. 
Proof. Each $\psi_{j, 0}$ is a $j$ th-dilation of $\psi \in W_{0}$. Therefore we must have that

$$
D^{j} \psi \in D^{j}\left(W_{0}\right)=D^{j}\left(V_{1} \ominus V_{0}\right)=D^{j}\left(V_{1}\right) \ominus D^{j}\left(V_{0}\right)=V_{j+1} \ominus V_{j}=W_{j}
$$

Any $\psi_{j, k}$ is just a translation of such a function, which does not affect the space the function resides in.

By taking enough translates of the dilations of $\psi$, we can span the $j$ th orthogonal complement $W_{j}$.

Lemma 3.3.9. The functions $\left\{\psi_{j, k}: 0 \leq k \leq 2^{j}-1\right\}$ span $W_{j}$.

Proof. This follows from Lemma 3.3.4; if we iterate that commutation relation we obtain

$$
T \circ D^{j}=D^{j} \circ T^{2^{j}}
$$

Thus only translates from 0 up to $2^{j}-1$ are required to span $W_{j}$.

Putting all the translations and dilations together gives the following theorem.

Theorem 3.3.10. Let $X=\{\phi\} \cup\left\{\psi_{j, k}: j \in \mathbb{Z}_{\geq 0}, 0 \leq k \leq 2^{j}-1\right\}$. Then $X$ forms a countable module basis for $\Xi$.

Proof. Since $\phi$ spans $V_{0}$ and for fixed $j,\left\{\psi_{j, k}\right\}$ span $W_{j}$, the collection $X$ is enough to span the entire union $\cup_{j=0}^{\infty} V_{j}$, and due to the density condition of the PMRA is enough to span $\Xi$.

That the basis is countable is easily seen by the enumeration

$$
\phi \mapsto 1, \psi_{j, k} \mapsto 2^{j}+k+1
$$

\subsubsection{Isomorphism with $l^{2}\left(A_{\alpha}\right)$}

The basis constructed in the previous section is a countable module basis for $\Xi$. This fact allows us to give a different proof of the following fact, first proved by M. Rieffel in [16]. 
Theorem 3.3.11. $\Xi$ is isomorphic to $l^{2}\left(A_{\alpha}\right)$ as Hilbert $A_{\alpha}$-modules.

Proof. Referring back to Example 2.2.12 with $A=A_{\alpha}$, it is clear to see that similar to the standard Hilbert space $l^{2}, l^{2}\left(A_{\alpha}\right)$ has a countable module basis $\left\{e_{i}\right\}_{i=1}^{\infty}$ with

$$
e_{i}=(0,0, \ldots, 1,0,0, \ldots)
$$

where the 1 is in the $i$ th component.

However, the module basis constructed for $\Xi$ is also a countable basis, and thus we can map that basis to the module basis for $l^{2}\left(A_{\alpha}\right)$ via whatever enumeration we want. For example, if we consider the wavelet $\psi \in W_{0}$ to be $\psi_{0,0}$, then one such enumeration yields the mapping

$$
\begin{gathered}
\phi \mapsto e_{1} \\
\psi_{j, k} \mapsto e_{2^{j}+k+1}
\end{gathered}
$$

This maps the module basis for $\Xi$ onto the module basis for $l^{2}\left(A_{\alpha}\right)$ and therefore gives us a concrete isomorphism between the two $A_{\alpha}$-modules $\Xi$ and $l^{2}\left(A_{\alpha}\right)$. 


\section{Chapter 4}

\section{Other Examples of PMRAs}

In this chapter we will examine basic ways of extending our example to form additional examples of PMRAs over noncommutative $\mathrm{C}^{\star}$-algebras. These methods will all result in PMRAs whose initial modules are free; we will consider other methods in the subsequent chapter.

\subsection{Compact Spaces $Y$}

Our first projective multiresolution analysis employs the Morita equivalence from Example 2.3.19 under the specific case where $Y=\mathbb{T}$ and the homeomorphism $T: \mathbb{T} \rightarrow \mathbb{T}$ is defined by

$$
T(z)=e^{2 \pi i \alpha} z
$$

for an irrational $0<\alpha<1$. However, there is no reason we have to choose this space and homeomorphism; we can attempt to let our same construction follow through for any compact space $Y$ and any homeomorphism $T: Y \rightarrow Y$.

Remark 4.1.1. In the proof of Theorem 3.2.3, one complication that arose was the use of the conditional expectation $E$ to show that the intersection of the $V_{i}$ was trivial. This required the integration of functions from $C(\mathbb{T})$ over the circle to yield complex coefficients. Thus, if we want to generalize this argument, we must require our compact space to have a finite, invariant measure $\nu$ against which to integrate functions in $C(Y)$.

With this adjustment, we can construct a similar PMRA for the more general example: 
Theorem 4.1.2. Let $Y$ be a compact space equipped with an finite, invariant measure $\nu$, let $T$ : $Y \rightarrow Y$ be a homeomorphism, and suppose $M$ is the mapping torus for $Y$, that is, $M=Y \times \mathbb{R} / \sim$, where the equivalence is given by $(y, r) \sim(T y, r+1)$. Define $\Lambda$ to be the completion of $C_{c}(Y \times \mathbb{R})$ under the norm induced by the $C_{c}(Y \times \mathbb{Z})$-valued inner product

$$
C_{c}(Y \times \mathbb{Z})\left\langle\Psi_{1}, \Psi_{2}\right\rangle(y, n)=\int_{\mathbb{R}} \Psi_{1}(y, t) \overline{\Psi_{2}\left(T^{n} y, t-n\right)} d t .
$$

Then $\Lambda$ can be given a left $(C(Y) \times \mathbb{Z})-P M R A$ structure, where the transformation algebra $C(Y) \times \mathbb{Z}$ is the completion of $C_{c}(Y \times \mathbb{Z})$ under the same norm.

Proof. Our construction will mirror that of Theorem 3.2.3, and the proof will be largely the same. First we consider a scaling function $\phi$, which must also satisfy

$$
C(Y) \times \mathbb{Z}\langle\phi, \phi\rangle=i d_{C(Y) \times \mathbb{Z}}
$$

or

$$
C(Y) \times \mathbb{Z}\langle\phi, \phi\rangle(y, n)=\int_{\mathbb{R}} \phi(y, r) \overline{\phi\left(T^{n} y, r-n\right)} d r=\delta(0, n)
$$

As before, we will choose $\phi$ to ignore the first variable, making it only dependent on the real variable. That is, we pick

$$
\phi(y, r)=\phi(r)
$$

and that reduces our requirement as before to

$$
\int_{\mathbb{R}} \phi(r) \overline{\phi(r-n)} d r=\delta(0, n)
$$

We then must choose a $\phi \in V_{0}$ carefully so that $\phi \in V_{1}$ as well; since we choose $\phi$ to be independent of the compact space variable $y$, this argument proceeds just as before to yield that $\phi$ must satisfy Equation 3.2:

$$
\phi(r)=\sum_{j \in \mathbb{Z}} c_{j} \phi(2 r-j) \text { for finitely many nonzero constants } c_{j}
$$

We can again pick a scaling function such as that of Example 3.2.2, or a similar function, and set our $\phi$ equal to that. 
The densely-defined $D: C_{c}(Y \times \mathbb{R}) \rightarrow C_{c}(Y \times \mathbb{R})$ can be defined analogously as well:

$$
D \Psi(y, r)=\sqrt{2} \Psi(y, 2 r)
$$

where we again only dilate in the real variable and leave the compact space variable alone. We then define $V_{0}=(C(Y) \times \mathbb{Z}) \cdot \phi$, and for any $j \in \mathbb{Z}$ define $V_{j}=\overline{D^{j}\left(C_{c}(Y \times \mathbb{Z}) \cdot \phi\right)}$. This yields our family $\left\{V_{i}\right\} \subset \Lambda$, and we again must verify the four conditions of Definition 3.1.4; the proof will largely parallel that of Theorem 3.2.3.

(i) $V_{0}$ is a projective $(C(Y) \times \mathbb{Z})$-submodule of $\Lambda$ : as before, since $V_{0}=(C(Y) \times \mathbb{Z}) \cdot \phi$, it is in fact a singly-generated free submodule of $\Lambda$.

(ii) For all $j, V_{j-1} \subset V_{j}$ : The scaling function $\phi$ was chosen so that $\phi \in V_{1}$, which implies that $V_{0} \subset V_{1}$. Applying powers of the densely defined $D$ and Equation 3.2 to that relation shows that $V_{j-1} \subset V_{j}$ for all $j \in \mathbb{Z}$.

(iii) $\cup_{j}^{\infty} V_{j}$ is dense in $\Xi$ : To prove the density condition, it will be enough to show that $C_{c}(Y \times \mathbb{R})$ is contained in the closure of this union. We will reuse Lemma 3.2.4 in the slightly more general context; the proof is exactly the same.

Lemma 4.1.3. Viewed as a subspace of $C_{c}(Y \times \mathbb{R}), C_{c}(\mathbb{R}) \subset \overline{\bigcup_{j=0}^{\infty} V_{j}}$.

Now, given any $g \in C(Y)$, define $M_{g} \in C_{c}(Y \times \mathbb{Z})$ to be given by

$$
M_{g}(y, n)= \begin{cases}g(y) & n=0 \\ 0 & n \neq 0\end{cases}
$$

Then given any $\gamma \in C_{c}(\mathbb{R}) \subset C_{c}(Y \times \mathbb{R})$, applying the action from the Hilbert $(C(Y) \times \mathbb{Z})$ module structure gives us

$$
M_{g} \cdot \gamma(y, r)=\sum_{j \in \mathbb{Z}} M_{g}(y, j) \gamma\left(T^{j} y, r-j\right)=g(y) \gamma(r)
$$


This combined with Lemma 4.1.3 implies that

$$
g(y) \gamma(r) \in \overline{\bigcup_{j=0}^{\infty} V_{j}}
$$

As before, this extends to any finite sum of such $g(y) \gamma(r)$, and since such sums can be used to approximate all elements of $C_{c}(Y \times \mathbb{R})$ in the uniform norm, we get that

$$
C_{c}(Y \times \mathbb{R}) \subset \overline{\bigcup_{j=0}^{\infty} V_{j}}
$$

as desired.

(iv) $\cap_{-\infty}^{\infty} V_{j}=\{0\}$ : We again use a similar argument, modified for our more general setting.

Lemma 4.1.4. Let $V_{0}=(C(Y) \times \mathbb{Z}) \cdot \phi$ be an $(C(Y) \times \mathbb{Z})$-submodule of $\Lambda$, and set $V_{j}=D^{j}\left(V_{0}\right)$ for all $j$. Then

$$
\bigcap_{-\infty}^{\infty} V_{j}=\{0\}
$$

Proof. Let $\xi \in \cap_{-\infty}^{\infty} V_{j}$. Then in particular, $\xi \in V_{0}=(C(Y) \times \mathbb{Z}) \cdot \phi$, so we can write $\xi=f_{\xi} \cdot \phi$ for some $f_{\xi}$ in $C(Y) \times \mathbb{Z}$. Therefore $f_{\xi} \cdot \phi \in V_{j}$ for all $j \in \mathbb{Z}$.

Now consider for a fixed $g \in C(Y)$ the multiplication operator $M_{g}$ defined above. A simple calculation shows that $M_{g}$ commutes with $D$; for any $\Psi \in C_{c}(Y \times \mathbb{R})$ :

$$
\begin{aligned}
& D\left(M_{g} \cdot \Psi(y, r)\right)=D(g(y) \Psi(y, r))=\sqrt{2} g(y) \Psi(y, 2 r) \\
& =g(y)(\sqrt{2} \Psi(y, 2 r))=g(y) D \Psi(y, r)=M_{g} \cdot D \Psi(y, r)
\end{aligned}
$$

Thus since $f_{\xi} \cdot \phi \in V_{j}$ for all $j \in \mathbb{Z}$, we have for any $g \in C(Y)$ that

$$
M_{g} f_{\xi} \cdot \phi \in M_{g} V_{j}=V_{j}
$$

since $V_{j}$ is closed under multiplication by $g$ as in Theorem 3.2.3.

Now $\mathbb{C} \times{ }_{\alpha} \mathbb{Z}$ is a subalgebra of $C(Y) \times \mathbb{Z}$, consisting of functions of the form

$$
\sum_{n \in \mathbb{Z}} c_{n} U^{n}
$$


for complex-valued constants $c_{n}$. We will consider the conditional expectation $E: C(Y) \times$ $\mathbb{Z} \rightarrow \mathbb{C} \times_{\alpha} \mathbb{Z}$ given by

$$
E\left(\sum_{n \in \mathbb{Z}} f_{n}(y) U^{n}\right)=\sum_{n \in \mathbb{Z}}\left(\int_{Y} f_{n}(y) d \nu\right) U^{n}
$$

in other words, the constant coefficients are obtained by integrating the functions $f_{n}(y) \epsilon$ $C(Y)$ with respect to the invariant measure $\nu$; since $\nu$ is finite and invariant, this will always yield a complex number.

Since $\mathbb{C} \times{ }_{\alpha} \mathbb{Z}$ is a subalgebra of $C(Y) \times \mathbb{Z}$ and $V_{0}=(C(Y) \times \mathbb{Z}) \cdot \phi$, for any $f \in C(Y) \times \mathbb{Z}$ we have that

$$
E(f) \cdot \phi \in V_{0}
$$

Recall that for all $j \in \mathbb{Z}$,

$$
V_{j}=\overline{\left\{\sum_{k \in \mathbb{Z}}(\sqrt{2})^{j} f(y, k) \phi\left(2^{j} r-k\right): f \in C_{c}(Y \times \mathbb{Z})\right\}}
$$

Since $\phi$ can be expressed as a finite linear combination of functions of the form

$$
\left\{(\sqrt{2})^{j} \phi\left(2^{j} t-k\right): k \in \mathbb{Z}\right\}
$$

for any $f \in C_{c}(Y \times \mathbb{Z})$, we have for any $j$,

$$
E(f) \cdot \phi \in V_{j}
$$

Therefore by a density argument and the continuity of $E$, we get $E\left(f_{\xi}\right) \cdot \phi \in V_{j}$ for all $j \in \mathbb{Z}$. If we let $f_{\xi}=\sum_{n \in \mathbb{Z}} \xi_{n}(t) U^{n}$, then this implies that

$$
\int_{Y} \xi_{n}(y) d \nu=0 \text { for all } n \in \mathbb{Z}
$$

But if we now recall Equation 4.1, the same reasoning applied to $M_{g} f_{\xi}$ shows that for any $g \in C(Y)$,

$$
\int_{Y} g(y) \xi_{n}(y) d \nu=0 \text { for all } n \in \mathbb{Z}
$$


which is enough to show that $\xi_{n}(y)=0$ for all $n \in \mathbb{Z}$. Hence $f_{\xi}$ is identically 0 , and $\xi=f_{\xi} \cdot \phi=0$ as well, and the intersection of the $V_{j}$ is trivial.

Thus all four conditions in Definition 3.1.4 are satsified, and the family $\left\{V_{j}\right\}$ forms a projective multiresolution analysis for $\Lambda$ over $C(Y) \times \mathbb{Z}$.

Given this construction, we can look at the following example.

Corollary 4.1.5. Let $Y=\mathbb{T}^{n}$, the $n$-dimensional torus, and let $T: \mathbb{T}^{n} \rightarrow \mathbb{T}^{n}$ be the homeomorphism

$$
T\left(z_{1}, z_{2}, \ldots, z_{n}\right)=\left(e^{2 \pi i \alpha_{1}} z_{1}, e^{2 \pi i \alpha_{2}} z_{2}, \ldots, e^{2 \pi i \alpha_{n}} z_{n}\right),
$$

where the $\alpha_{i}$ are irrational numbers between 0 and 1 . Then there is a left $C\left(\mathbb{T}^{n}\right) \times \mathbb{Z}-P M R A$ structure for the completion of $C\left(\mathbb{T}^{n} \times \mathbb{R}\right)$.

Proof. $Y=\mathbb{T}^{n}$ is a compact Hausdorff topological group, and hence there exists a Haar measure $\nu$ on $\mathbb{T}^{n}$ for which $\nu\left(\mathbb{T}^{n}\right)<\infty$. We can construct the Morita equivalence of Example 2.3.19, and then Theorem 4.1.2 applies to give our PMRA structure.

\subsection{Direct Sums of PMRAs}

A fruitful way to create new examples of PMRAs over noncommutative $\mathrm{C}^{*}$-algebras is to find ways of combining two PMRAs with compatible structures into a single PMRA structure for a more complicated object. We will consider two such ways: direct sums and tensor products, which we will delay to the next chapter. For now, let us consider direct sums.

Theorem 4.2.1. Let $A$ be a $C^{*}$-algebra, and suppose that $\Xi$ and $\Omega$ are both left (or right) Hilbert A-modules. Suppose further that the families of subspaces $\left\{V_{i}\right\}$ and $\left\{W_{i}\right\}$ are PMRAs for $\Xi$ and $\Omega$, respectively. Then the family of subspaces $\left\{Y_{i}\right\}$ given by $Y_{i}=V_{i} \oplus W_{i}$ forms a left (or right) A-PMRA structure for the Hilbert module $\Xi \oplus \Omega$. 
Proof. Proposition 2.2.10 shows that $\Xi \oplus \Omega$ is in fact a left $A$-module. To show $\left\{Y_{i}\right\}$ forms a left $A$-PMRA structure, we must verify the four conditions of Definition 3.1.4.

(i) Since $V_{0}$ and $W_{0}$ are both $A$-modules, Proposition 2.2 .10 gives us that $Y_{0}$ is also an $A$ module. It is a submodule of $\Xi \oplus \Omega$ because $V_{0}$ is a submodule of $\Xi$ and $W_{0}$ is a submodule of $\Omega$, and since $V_{0}$ and $W_{0}$ are both projective, their direct sum is also the direct summand of a free module, hence $Y_{0}$ is projective as well.

(ii) Let $\left(v_{i}, w_{i}\right) \in Y_{i}$. Then $v_{i} \in V_{i} \subset V_{i+1}$, since the $V_{i}$ form a PMRA for $\Xi$. Similarly, $w_{i} \in W_{i} \subset$ $W_{i+1}$, and thus $\left(v_{i}, w_{i}\right) \in V_{i+1} \oplus W_{i+1}=Y_{i+1}$. Thus we have $Y_{i} \subset Y_{i+1}$.

(iii) Let $(\xi, \omega) \in \Xi \oplus \Omega$. Since $\xi \in \Xi$ and $\cup V_{i}$ is dense in $\Xi$, there is a sequence $\left\{v_{i}\right\} \subset \cup V_{i}$ such that $v_{i} \rightarrow \xi$ in the norm induced by the $A$-valued inner product on $\Xi$. Similarly, there exists a sequence $\left\{w_{i}\right\} \subset \bigcup W_{i}$ such that $w_{i} \rightarrow \omega$ in the norm induced by the $A$-valued inner product on $\Omega$. But then

$$
{ }_{A}\left\langle\left(v_{i}, w_{i}\right)-(\xi, \omega)\right\rangle={ }_{A}\left\langle v_{i}-\xi, v_{i}-\xi\right\rangle+{ }_{A}\left\langle w_{i}-\omega, w_{i}-\omega\right\rangle
$$

and thus as $i \rightarrow \infty$

$$
{ }_{A}\left\langle\left(v_{i}, w_{i}\right)-(\xi, \omega)\right\rangle \rightarrow 0+0=0
$$

Therefore, $\left(v_{i}, w_{i}\right) \rightarrow(\xi, \omega)$ and since $\left\{\left(v_{i}, w_{i}\right)\right\} \subset \cup Y_{i}$, we get that $\cup Y_{i}$ is dense in $\Xi \oplus \Omega$.

(iv) $\cap Y_{i}=\bigcap\left(V_{i} \oplus W_{i}\right)=\bigcap V_{i} \oplus \bigcap W_{i}=0 \oplus 0=0$

Since all four conditions are satisfied, we have that $\left\{Y_{i}\right\}$ is a left $A$-PMRA structure for $\Xi \oplus \Omega$.

In a similar fashion, we can construct direct sums of up to $n$ PMRA structures, provided they have the same underlying $\mathrm{C}^{*}$-algebra. As an example, let us consider summing a PMRA with itself. 
Corollary 4.2.2. Let $\Xi$ have the PMRA structure $\left\{V_{i}\right\}$ defined in Theorem 3.2.3. Define $\Xi^{n}$ to be $\Xi$ summed with itself $n$ times, and $V_{i}^{n}$ to be $V_{i}$ summed with itself $n$ times. Then $\left\{V_{i}^{n}\right\}$ forms a left $A_{\alpha}-P M R A$ structure for $\Xi^{n}$.

Remark 4.2.3. As in the compact space example, this is a projective multiresolution analysis where the initial module is free; while it is no longer singly generated, it is finitely generated by elements of the form

$$
0 \oplus 0 \oplus \ldots \oplus \phi_{k} \oplus \ldots \oplus 0
$$

where $\phi_{k}$ is the scaling function chosen for the $k$-th PMRA structure.

We hope now to create examples of PMRAs where the initial module is projective but not free; in order to do so, we will explore the tensor product of a PMRA structure. 


\section{Chapter 5}

\section{Tensor Products of PMRAs}

The examples in the previous chapter all share the property that the initial module of our PRMAs is not only a projective submodule, but also a free, finitely generated module. Our goal for this chapter is to produce examples of PMRAs whose initial modules are projective but not free, and our tactic will be to use tensor products of Hilbert modules.

\subsection{Tensor Products of PMRAs}

Remark 5.1.1. For convenience, we will refer to projective multiresolution analyses composed of left Hilbert $A$-modules as left $A$-PMRAs, and those composed of right Hilbert $A$-modules as right $A$-PMRAs. We also recall that $X \odot Y$ denotes the algebraic tensor product, $X \odot_{B} Y$ denotes the balanced tensor product, and $X \otimes_{B} Y$ denotes the internal tensor product.

We would like to be able to construct new examples of such PMRAs by tensoring with

other PMRA structures. Recall Proposition 2.3.13 tells us that, given two Morita equivalence structures, we can form the balanced tensor product of the equivalence bimodules and construct a Morita equivalence implemented by that balanced tensor product. In addition, our projective multiresolution analyses constructed in both Theorem 3.2.3 and Theorem 4.1.2 originated from a Morita equivalence structure, although the equivalence itself was not explicitly used. The goal will be to combine a PMRA constructed from a Morita equivalence with a second, compatible Morita equivalence, and use balanced tensor products to create a PMRA for the new equivalence bimodule. 
Thus, let us consider the following situation: suppose we have a $B$ - $C$ equivalence bimodule $\Xi$; that is, $\Xi$ is a full left Hilbert $B$-module and a full right Hilbert $C$-module. Furthermore, suppose that we have constructed a left $B$-PMRA structure $\left\{V_{i}\right\}$ for the left Hilbert $B$-module $\Xi$. Lastly, suppose we have an $A$ - $B$ equivalence bimodule $X$ that implements a Morita equivalence between $A$ and $B$. We can create the $B$-balanced tensor product $X \odot_{B} \Xi$ as in Definition 2.3.12, which by Proposition 2.3.13 implements a pre-equivalence structure between $A$ and $C$. The hope is that the left $B$-PMRA structure on $\Xi$ can be extended to a PMRA structure on the internal tensor product $X \otimes_{B} \Xi$

One complication that will arise in this construction is the lack of submodules; that is, for $j<0, V_{j}$ is not generally a submodule of $\Xi$, and thus constructing the balanced tensor product is not feasible. We will avoid this complication via the following definition.

Definition 5.1.2. Let $A$ be a $\mathrm{C}^{*}$-algebra with unit and let $\Xi$ be a countably generated (left) $A$-module. A quasi projective multiresolution analysis (QPMRA) for $\Xi$ over $A$ is a family $\left.\left\{V_{j}\right\}_{j \geq 0}\right\}$ of closed $A$-submodules of $\Xi$ such that

(i) $V_{0}$ is a projective $A_{\alpha}$-submodule of $\Xi$

(ii) $V_{j} \supset V_{j-1}$ for all $j \geq 1$,

(iii) $\cup_{j=0}^{\infty} V_{j}$ is dense in $\Xi$

Remark 5.1.3. A QPMRA, then, is a PMRA where all subspaces are $A$-submodules of $\Xi$; however, we have dropped the closed subspaces for negative indicies $j$ and similarly dropped the trivial intersection condition.

Given this definition, we now proceed with our tensor product construction.

Theorem 5.1.4. Let $A, B, C, X$, and $\Xi$ be defined and structured as above, and suppose in addition that the left B-PMRA $\left\{V_{i}\right\}$ consists of finitely generated $B$-modules, and that the $C^{*}$-algebra $A$ is unital. Define for all $i \geq 0$

$$
Z_{i}=X \otimes_{B} V_{i}
$$


Then $\left\{Z_{i}\right\}_{i \geq 0}$ is a left $A$-QPMRA structure for the left Hilbert A-module $X \otimes_{B} \Xi$.

Remark 5.1.5. We note that since $X \otimes_{B} \Xi$ is a Hilbert $A$-module, it naturally follows that the extended PMRA structure for this module is no longer over the $\mathrm{C}^{*}$-algebra $B$, but swaps itself to be over the $\mathrm{C}^{*}$-algebra $A$ instead. In addition, while the theorem is stated for left PMRAs and modules, there is an equivalent formulation involving right PMRAs and modules. In fact, given a left $A$-PMRA and a $A$ - $B$ equivalence bimodule $X$, we can use Example 2.3.10 to obtain a $B$ - $A$ equivalence bimodule over the same space $X$ and then apply the theorem to those two structures.

Proof. We must address just the three conditions of a QPMRA structure as given in Definition 5.1 .2 .

(i) Proposition 2.3.13 shows that $Z=X \otimes_{B} \Xi$ is an $A-C$ equivalence bimodule. We must show that $Z_{0}=X \otimes_{B} V_{0}$ is projective; our argument will involve finding a projection in $M_{n}(C)$, the matrix algebra over $C$. The argument will follow that of Rieffel's in [17].

Since $A$ is unital, the range of ${ }_{A}\langle\cdot, \cdot\rangle$, which is dense in $A$ since $X$ is a full left Hilbert $A$-module, must in fact coincide with $A$. Thus we can find elements $\left\{x_{i}\right\}_{i=1}^{n}$ and $\left\{y_{i}\right\}_{i=1}^{n}$ in $Z$ such that

$$
\sum_{i=1}^{n}{ }_{A}\left\langle x_{i}, y_{i}\right\rangle=1_{A}
$$

Let $M_{n}(C)$ be the matrix algebra over $C ; Z^{n}$ is then a $A-M_{n}(C)$ equivalence bimodule, and $x=\left\{x_{1}, x_{2}, \ldots, x_{n}\right\}$ and $y=\left\{y_{1}, y_{2}, \ldots, y_{n}\right\}$ are elements of $Z^{n}$ such that ${ }_{A}\langle x, y\rangle=1_{A}=$ ${ }_{A}\langle y, x\rangle$. Then we calculate:

$$
1={ }_{A}\langle x, y\rangle_{A}\langle y, x\rangle={ }_{A}\left\langle{ }_{A}\langle x, y\rangle \cdot y, x\right\rangle={ }_{A}\left\langle x \cdot\langle y, y\rangle_{M_{n}(C)}, x\right\rangle={ }_{A}\langle z, z\rangle
$$

where $z=x \cdot\langle y, y\rangle_{M_{n}(C)}^{1 / 2}$. Then

$$
\langle z, z\rangle_{M_{n}(C)}\langle z, z\rangle_{M_{n}(C)}=\left\langle z, z \cdot\langle z, z\rangle_{M_{n}(C)}\right\rangle_{M_{n}(C)}=\left\langle z{ }_{A}\langle z, z\rangle \cdot z\right\rangle_{M_{n}(C)}=\langle z, z\rangle_{M_{n}(C)}
$$

and therefore $p=\langle z, z\rangle_{M_{n}(C)}$ is a projection in $M_{n}(C)$. 
Define $\phi: A \rightarrow M_{n}(C)$ by $\phi(a)=\langle z, a \cdot z\rangle_{M_{n}(C)}$; this map is an injective *-homomorphism into the algebra $p M_{n}(C) p$. A straightforward calculation shows that, for any $x, y \in Z^{n}$,

$$
\begin{gathered}
\phi\left({ }_{A}\langle z, x\rangle_{A}\langle y, z\rangle\right)=\left\langle z,{ }_{A}\langle z, x\rangle_{A}\langle y, z\rangle \cdot z\right\rangle_{M_{n}(C)}=\left\langle{ }_{A}\langle x, z\rangle \cdot z,{ }_{A}\langle y, z\rangle \cdot z\right\rangle_{M_{n}(C)} \\
=\left\langle x \cdot\langle z, z\rangle_{M_{n}(C)}, y \cdot\langle z, z\rangle_{M_{n}(C)}\right\rangle_{M_{n}(C)}=\langle z, z\rangle_{M_{n}(C)}\langle x, y\rangle_{M_{n}(C)}\langle z, z\rangle_{M_{n}(C)} \\
=p\langle x, y\rangle_{M_{n}(C)} p
\end{gathered}
$$

Therefore the range of $\phi$ contains that of $p\langle\cdot, \cdot\rangle_{M_{n}(C)} p$. Since the range of $\langle\cdot, \cdot\rangle_{M_{n}(C)}$ is dense in $M_{n}(C)$, then the range of $p\langle\cdot, \cdot\rangle_{M_{n}(C)} p$ will be dense in $p M_{n}(C) p$. Therefore the range of $\phi$ is exactly $p M_{n}(C) p$, and $\phi$ is an isomorphism of $A$ onto $p M_{n}(C) p$.

Therefore, we can identify $Z^{n}$ with $p\left[\oplus_{i=1}^{n} C\right]$, which implies that we can write

$$
\bigoplus_{i=1}^{n} C=Z^{n} \oplus(1-p)\left[\bigoplus_{i=1}^{n} C\right]
$$

and therefore $Z^{n}$ is projective, hence $Z$ is projective.

Now since $V_{0}$ is finitely generated, it is also orthogonally complementable in $\Xi$; thus, there exists a projection $\pi$ such that $\pi(\Xi)=V_{0}$. Define the projection $q=\operatorname{id}_{X} \otimes_{B} \pi$; then $q$ takes $Z$ to $Z_{0}$. This gives us $Z=Z_{0} \oplus(1-q) Z$, and since $Z$ was projective, so is $Z_{0}$.

(ii) For the nesting condition, let $x \otimes_{B} v \in Z_{i}=X \otimes_{B} V_{i}$ be an elementary tensor. Since the $V_{i}$ form a PMRA for $\Xi$, we have that $V_{i} \subset V_{i+1}$, and hence $v \in V_{i+1}$. Thus any elementary tensor in $Z_{i}$ is also in $X \otimes_{B} V_{i+1}=Z_{i+1}$, and since the internal tensor product is a completion of the span of such elementary tensors, we have $Z_{i} \subset Z_{i+1}$.

(iii) We now return to the density condition and consider any elementary tensor $x \otimes \xi \in X \odot \Xi$, the algebraic tensor product. Since $\xi \in \Xi$ and $\left\{V_{i}\right\}$ is a left $B$-PMRA for $\Xi$, there exists a sequence of elements $v_{j} \in \cup V_{i}$ such that

$$
{ }_{B}\left\langle\xi-v_{j}, \xi-v_{j}\right\rangle \rightarrow 0
$$


We consider now the sequence of elements $z_{j}=x \otimes v_{j}$, and calculate via the definition of the inner product on the balanced tensor product:

$$
{ }_{A}\left\langle\left\langle x \otimes \xi-x \otimes v_{j}, x \otimes \xi-x \otimes v_{j}\right\rangle\right\rangle={ }_{A}\left\langle x, x \cdot{ }_{B}\left\langle\xi-v_{j}, \xi-v_{j}\right\rangle\right\rangle
$$

As $j \rightarrow \infty$, we get that

$$
{ }_{A}\left\langle\left\langle x \otimes \xi-x \otimes v_{j}, x \otimes \xi-x \otimes v_{j}\right\rangle\right\rangle \rightarrow{ }_{A}\langle x, x \cdot 0\rangle=0
$$

Thus any elementary tensor in $X \odot \Xi$ can be approximated by elements of $\cup Z_{i}$. Now fix an arbitrary tensor in the algebraic tensor product $X \odot \Xi$ :

$$
\delta=\sum_{i=1}^{n}\left(x_{i} \otimes \xi_{i}\right)
$$

Let $\epsilon>0$. For all $1 \leq i \leq n$, there exists a sequence $\left\{x_{i} \otimes v_{i, j}\right\}$ and a number $N_{i}$ such that for all $j \geq N_{j}$,

$$
{ }_{A}\left\langle\langle x _ { i } \otimes \xi _ { i } - x _ { i } \otimes v _ { i , j } \rangle \left\langle<\frac{\epsilon}{n} .\right.\right.
$$

Define $\delta_{j}=\sum_{i=1}^{n}\left(x_{i} \otimes v_{i, j}\right)$ for each $j$; we calculate:

$$
\begin{aligned}
{ }_{A}\left\langle\left\langle\delta-\delta_{j}, \delta-\delta_{j}\right\rangle\right\rangle & =\sum_{i, k}{ }_{A}\left\langle\left\langle\left(x_{i} \otimes \xi_{i}\right)-\left(x_{i} \otimes v_{i, j}\right),\left(x_{k} \otimes \xi_{k}\right)-\left(x_{k} \otimes v_{k, j}\right)\right\rangle\right\rangle \\
& =\sum_{i, k} A\left\langle x_{i}, x_{k} \cdot{ }_{B}\left\langle\xi_{k}-v_{k, j}, \xi_{i}-v_{i, j}\right\rangle\right\rangle
\end{aligned}
$$

Now let $N=\max _{j} N_{j}$; then for any $1 \leq i \leq n$, if $j>N$, then $\left\|_{B}\left\langle\xi_{i}-v_{i, j}, \xi_{i}-v_{i, j}\right\rangle\right\|<\frac{\epsilon}{n}$. Thus by applying Equation 2.3 to the $B$-valued inner product, we get

$$
\left\|_{B}\left\langle\xi_{k}-v_{k, j}, \xi_{i}-v_{i, j}\right\rangle\right\|^{2} \leq\left\|_{B}\left\langle\xi_{k}-v_{k, j}, \xi_{k}-v_{k, j}\right\rangle\right\|\left\|_{B}\left\langle\xi_{i}-v_{i, j}, \xi_{i}-v_{i, j}\right\rangle\right\|<\left(\frac{\epsilon}{n}\right)^{2}
$$

Thus as $j \rightarrow \infty,{ }_{B}\left\langle\xi_{k}-v_{k, j}, \xi_{i}-v_{i, j}\right\rangle \rightarrow 0$ for any pair $1 \leq i, k \leq n$, and applying this to 5.2 gives

$$
{ }_{A}\left\langle\left\langle\delta-\delta_{j}, \delta-\delta_{j}\right\rangle\right\rangle \rightarrow 0 \text { as } j \rightarrow 0
$$

Therefore any tensor in $X \odot \Xi$ can be approximated by elements of $\cup Z_{i}$. Since $X \otimes_{B} \Xi$ is a quotient of the algebraic tensor product, the same holds for any element of $X \otimes_{B} \Xi$, and hence $\cup Z_{i}$ is dense in $X \otimes_{B} \Xi$. 
Thus we have satisfied all conditions of Definition 5.1.2, and $\left\{Z_{i}\right\}$ is a left $A$-QPMRA for $X \otimes_{B} \Xi$

\subsection{Examples}

Here we will apply our theorem to create a few examples. These examples will all use the first example constructed in Chapter 3, and apply the tensor product construction using that example and some interesting Morita equivalence involving an irrational rotation algebra. Hence these examples will all be PMRAs over some $A_{\alpha}$, but due to the tensor product construction the initial modules will not be free.

\subsubsection{The Bimodule $V_{\alpha}(p, q)$}

First, while we know from Proposition 2.3.17 when $A_{\alpha}$ and $A_{\beta}$ are Morita equivalent, we have not specifically seen the implementation of this; we will invoke the following proposition, which is a special case of a theorem of Rieffel in [18].

Remark 5.2.1. We will denote by $\mathbb{Z}_{n}$ the cyclic group of order $n$, and $[k]_{n}$ will denote the image of the integer $k$ in this group.

Proposition 5.2.2. [18] Let $\alpha$ be an irrational number, and let

$$
\beta=\frac{a \alpha+b}{q \alpha+p} \text { for }\left(\begin{array}{ll}
a & b \\
q & p
\end{array}\right) \in G L(2, \mathbb{Z}) \text { and } q \neq 0 .
$$

Let $G=\mathbb{R} \times \mathbb{Z}_{q}$, and let $\gamma=(q \alpha+p)^{-1}$. Then $C_{c}(G)$ is a pre-equivalence $A_{\beta}-A_{\alpha}$ bimodule under the inner products

$$
\begin{gathered}
\langle f, g\rangle_{A_{\alpha}}(n, s)=\sum_{m} \bar{f}\left((q s-m) \gamma,[-m]_{q}\right) g\left((q s-m) \gamma+n,[p n-m]_{q}\right) \\
A_{\beta}\langle f, g\rangle\left(n,\left(r,[k]_{q}\right)\right)=\sum_{m} f\left(a k+q r+m,[k+p m]_{q}\right) \bar{g}\left(a k+q r+m-n \gamma,[k+p m-n]_{q}\right)
\end{gathered}
$$


Remark 5.2.3. We will follow Rieffel's notation in [18] and denote the completion of this module with respect to the $A_{\alpha}$-valued inner product as $V_{\alpha}(p, q)$.

We now construct a quasi projective multiresolution by taking our existing left $A_{\alpha}$-PMRA from Theorem 3.2.3 and using Theorem 5.1.4 to combine it with the Morita equivalence implemented by $V_{\alpha}(p, q)$.

Theorem 5.2.4. Let $\alpha$ be an irrational number between 0 and 1 , and let $\Xi$ and $\left\{V_{i}\right\}$ be the left $A_{\alpha}$-PMRA constructed in Theorem 3.2.3. Let $V_{\alpha}(p, q)$ be an $A_{\beta}-A_{\alpha}$ equivalence bimodule as in Proposition 5.2.2. Then the family of submodules $\left\{Z_{i}\right\}_{i=0}^{\infty}$ given by

$$
Z_{i}=V_{\alpha}(p, q) \otimes_{A_{\alpha}} V_{i}
$$

forms a left $A_{\beta}$-QPMRA structure for the left Hilbert $A_{\beta}$-module $V_{\alpha}(p, q) \otimes_{A_{\alpha}} \Xi$.

Proof. This is a direct application of Theorem 5.1.4, with $A=A_{\beta}, B=A_{\alpha}, C=C(M) \times \mathbb{R}$, $X=V_{\alpha}(p, q)$, and $\Xi$ the completion of $C_{c}(\mathbb{T} \times \mathbb{R})$ in the norm induced by the $A_{\alpha}$-valued inner product.

Remark 5.2.5. A particularly interesting special case of this construction is when $a=p=0$ and $b=q=1 ;$ in this case, $\beta=\frac{1}{\alpha}$, and we construct an $A_{\frac{1}{\alpha}}$-QPMRA for $V_{\alpha}(0,1) \otimes_{A_{\alpha}} \Xi$. Our next example will also employ a Morita equivalence between $A_{\frac{1}{\alpha}}$ and $A_{\alpha}$, albeit one implemented by a very different equivalence bimodule.

\subsubsection{Feichtinger's Algebra $S_{0}(\mathbb{R})$}

For our next example, we will employ a Morita equivalence which is a specific case of a construction found in Luef. In [10], he constructs a general Morita equivalence between the twisted $\mathrm{C}^{*}$-algebras $C^{*}(\Lambda, \beta)$ and $C^{*}\left(\Lambda^{0}, \bar{\beta}\right)$, where $\Lambda$ is a lattice in $\mathbb{R}^{d} \times \hat{\mathbb{R}}^{d}, \Lambda^{0}$ is the adjoint lattice, and $\beta$ is a 2-cocycle. By letting $\Lambda=\mathbb{Z}^{2 d}$, this reduces to an noncommutative $2 d$-torus. Here we will restrict our attention to the case $d=1$, which gives us the lattice $\Lambda=\mathbb{Z}^{2}$ and a noncommutative 2-torus, otherwise known as an irrational rotation algebra. 
We will state several definitions used in [10], give the formulation of the Morita equivalence, and then use it to construct a projective multiresolution analysis.

Definition 5.2.6. Let $\lambda=(x, w) \in \mathbb{R} \times \hat{\mathbb{R}}$. The time-frequency shift $\pi(\lambda)$ is defined to be

$$
\pi(\lambda)(f)=e^{2 \pi i w t} f(t-x)
$$

that is, translation by $x \in \mathbb{R}$ and modulation by $w \in \hat{R}$.

Remark 5.2.7. Following Luef, we can use time-frequency shifts to represent the irrational rotation algebra on $L^{2}(\mathbb{R})$; when done this way, elements are of the form

$$
\sum_{\lambda \in \mathbb{Z}^{2}} a_{\lambda} \pi(\lambda)
$$

Definition 5.2.8. Let $f, g \in L^{2}(\mathbb{R})$. We define the short time Fourier transform (STFT) of $f$ with respect to $g$ to be

$$
V_{g} f(x, w)=\int_{\mathbb{R}} f(t) \overline{g(t-x)} e^{-2 \pi i t w} d t
$$

for $(x, w) \in \mathbb{R} \times \hat{\mathbb{R}}$.

Definition 5.2.9. We define Feichtinger's algebra to be

$$
S_{0}(\mathbb{R})=\left\{f \in L^{2}(\mathbb{R}): \iint_{\mathbb{R} \times \hat{\mathbb{R}}}\left|V_{\phi} f(x, w)\right| d x d w<\infty\right\}
$$

where $V_{\phi} f$ is the STFT of $f$ with respect to the window function $\phi(x)=2^{-\frac{1}{4}} e^{-x^{2}}$.

Proposition 5.2.10. [10] $S_{0}(\mathbb{R})$ implements a Morita equivalence between $A_{\alpha}$ and $A_{\frac{1}{\alpha}}$ via the actions

$$
a \cdot g=\sum_{\lambda \in \mathbb{Z}^{2}} a_{\lambda} \pi(\lambda) g \quad g \cdot b=\sum_{\lambda \in \mathbb{Z}^{2}} b_{\lambda} \pi^{*}(\lambda) g
$$

for $a \in A_{\alpha}, b \in A_{\frac{1}{\alpha}}$, and $g \in S_{0}(\mathbb{R})$, and inner products

$$
A_{\alpha}\langle f, g\rangle=\sum_{\lambda \in \mathbb{Z}^{2}} V_{g} f(\lambda) \pi(\lambda) \quad\langle f, g\rangle_{A_{\frac{1}{\alpha}}}=\sum_{\lambda \in \mathbb{Z}^{2}}\langle\pi(\lambda) g, f\rangle \pi(\lambda)
$$

for $f, g \in S_{0}(\mathbb{R})$. 
As with our previous example, we use the PMRA constructed in Chapter 3 and combine it with the Morita equivalence implemented by $S_{0}(\mathbb{R})$ using Theorem 5.1.4.

Theorem 5.2.11. There exists a left $A_{\alpha}-Q P M R A$ structure for the left $A_{\alpha}$-module $S_{0}(\mathbb{R}) \otimes_{A_{\frac{1}{\alpha}}} \Xi$, where $\Xi$ is the completion of $C(\mathbb{T} \times \mathbb{R})$ with respect to the norm induced by the $A_{\frac{1}{\alpha}}$-valued inner product.

Proof. By repeating our construction in Chapter 3 with $\frac{1}{\alpha}$ in place of $\alpha$, we construct a left $A_{\frac{1}{\alpha}}{ }^{-}$ PMRA structure $\left\{V_{i}\right\}$ for the left $A_{\frac{1}{\alpha}}$-module $\Xi$. Combining this with Theorem 5.1.4 and the Morita equivalence from Proposition 5.2.10, we obtain the left $A_{\alpha}$-QPMRA $\left\{Z_{i}\right\}_{i=0}^{\infty}$ for $S_{0}(\mathbb{R}) \otimes_{A_{\frac{1}{\alpha}}} \Xi$, where for $i \geq 0$,

$$
Z_{i}=S_{0}(\mathbb{R}) \otimes_{A_{\frac{1}{\alpha}}} V_{i}
$$

\subsection{Module Frames}

In this section, we'll seek to extend the module frame in our existing projective multiresolution analysis to the PMRA formed via the tensor product construction. The key part will be to find a module frame for our bimodule $X$, which we acheive through the following lemma.

Lemma 5.3.1. Let $\alpha$ be an irrational number between 0 and 1 . If $X$ is an $A_{\beta}-A_{\alpha}$ equivalence bimodule, then there exist functions $\left\{f_{i}\right\}$ in $X$ such that, for all $x \in X$,

$$
A_{\beta}\langle x, x\rangle=\sum_{i=1}^{n} A_{\beta}\left\langle x, f_{i}\right\rangle_{A_{\beta}}\left\langle f_{i}, x\right\rangle
$$

Proof. Since $A_{\alpha}$ is unital, there exist functions $f_{i}$ such that

$$
\sum_{i=1}^{n}\left\langle f_{i}, f_{i}\right\rangle_{A_{\alpha}}=\mathrm{id}_{A_{\alpha}}
$$

We then calculate

$$
A_{\beta}\langle x, x\rangle={ }_{A_{\beta}}\left\langle x \cdot \sum_{i=1}^{n}\left\langle f_{i}, f_{i}\right\rangle_{A_{\alpha}}, x\right\rangle=\sum_{i=1}^{n}{ }_{A_{\beta}}\left\langle A_{\beta}\left\langle x, f_{i}\right\rangle \cdot f_{i}, x\right\rangle=\sum_{i=1}^{n}{ }_{A_{\beta}}\left\langle x, f_{i}\right\rangle_{A_{\beta}}\left\langle f_{i}, x\right\rangle .
$$


In other words, the functions $f_{i}$ form a finite standard module frame for $X$ as an $A_{\beta}$-module. We now seek a way of combining two module frames.

Theorem 5.3.2. Let $X$ be an $A-B$ equivalence bimodule, let $Y$ be a $B-C$ equivalence bimodule, and let $Z=X \otimes_{B} Y$. If $X$ has a countable module frame $\left\{f_{i}\right\}$ as a left A-module and $Y$ has a countable module frame $\left\{g_{i}\right\}$ as a left B-module, then $\left\{f_{i} \otimes g_{j}\right\}_{i, j}$ is a countable module frame for $Z$ as a left $A$-module.

Proof. First, we note that since $\left\{f_{i}\right\}$ and $\left\{g_{i}\right\}$ are both countable, so is $\left\{f_{i} \otimes g_{j}\right\}$. We want to show that, for any simple tensor $x \otimes y \in Z$,

$$
{ }_{A}\left\langle\langle x \otimes y, x \otimes y\rangle=\sum_{i, j}{ }_{A}\left\langle\langle x \otimes y , f _ { i } \otimes g _ { j } \rangle _ { A } \left\langle\left\langle f_{i} \otimes g_{j}, x \otimes y\right\rangle .\right.\right.\right.
$$

We calculate using the definition of the $A$-valued inner product on $Z$ :

$$
{ }_{A}\langle\langle x \otimes y, x \otimes y\rangle\rangle={ }_{A}\left\langle x, x \cdot{ }_{B}\langle y, y\rangle\right\rangle={ }_{A}\left\langle x, x \cdot\left(\sum_{j}{ }_{B}\left\langle y, g_{j}\right\rangle_{B}\left\langle g_{j}, y\right\rangle\right)\right\rangle
$$

where we apply the definition the module frame $\left\{g_{i}\right\}$,

$$
=\sum_{j}{ }_{A}\left\langle x, x \cdot{ }_{B}\left\langle y, g_{j}\right\rangle_{B}\left\langle g_{j}, y\right\rangle\right\rangle=\sum_{j}{ }_{A}\left\langle x \cdot{ }_{B}\left\langle y, g_{j}\right\rangle, x \cdot{ }_{B}\left\langle y, g_{j}\right\rangle\right\rangle
$$

Since $x \cdot{ }_{B}\left\langle y, g_{j}\right\rangle \in X$, we can apply the module frame $\left\{f_{i}\right\}$ to get

$$
\begin{aligned}
& =\sum_{j} \sum_{i}{ }_{A}\left\langle x \cdot{ }_{B}\left\langle y, g_{j}\right\rangle, f_{i}\right\rangle_{A}\left\langle f_{i}, x \cdot{ }_{B}\left\langle y, g_{j}\right\rangle\right\rangle \\
& =\sum_{j} \sum_{i}{ }_{A}\left\langle x, f_{i} \cdot{ }_{B}\left\langle g_{j}, y\right\rangle\right\rangle_{A}\left\langle f_{i}, x \cdot{ }_{B}\left\langle y, g_{j}\right\rangle\right\rangle \\
& =\sum_{i, j}{ }_{A}\left\langle\left\langle x \otimes y, f_{i} \otimes g_{j}\right\rangle\right\rangle_{A}\left\langle\left\langle f_{i} \otimes g_{j}, x \otimes y\right\rangle\right\rangle,
\end{aligned}
$$

as desired.

Since our examples in the previous section both stem from Morita equivalences between $A_{\alpha}$ and $A_{\beta}$, and our original projective multiresolution analysis for $\Xi$ yielded a countable standard module frame, we can combine our results to produce frames for our tensor product examples. 
Corollary 5.3.3. There exists a standard module frame for the left $A_{\beta}$-module $V_{\alpha}(p, q) \otimes_{A_{\alpha}} \Xi$.

Proof. By Lemma 5.3.1, there exists a (finite) standard module frame $\left\{f_{i}\right\}$ for $V_{\alpha}(p, q)$ as a left $A_{\beta^{-}}$ module. Let $\phi_{k}$ be the module frame (with an appropriate enumeration) for $\Xi$ found in Theorem 3.3.10. Then by Theorem 5.3.2, the family of functions $f_{i} \otimes_{A_{\alpha}} \phi_{k}$ forms a standard module frame for the module $V_{\alpha}(p, q) \otimes_{A_{\alpha}} \Xi$.

A similar result holds for the example from Theorem 5.2.11:

Corollary 5.3.4. There exists a standard module frame for the left $A_{\alpha}$-module $S_{0}(\mathbb{R}) \otimes_{A_{\frac{1}{\alpha}}} \Xi$.

Proof. By Lemma 5.3.1, there exists a finite standard module frame $\left\{g_{i}\right\}$ for $S_{0}(\mathbb{R})$ as a left $A_{\alpha^{-}}$ module. Let $\psi_{k}$ be a module frame (with an appropriate enumeration) for $\Xi$ as a left $A_{\frac{1}{\alpha}}$-module, analogous to that found in Theorem 3.3.10. Then by Theorem 5.3.2, the family of functions $g_{i} \otimes_{A_{\frac{1}{\alpha}}} \psi_{k}$ forms a standard module frame for the module $S_{0}(\mathbb{R}) \otimes_{A_{\frac{1}{\alpha}}} \Xi$. 


\section{Bibliography}

[1] A. Connes. Noncommutative geometry. Academic Press Inc., 1994.

[2] I. Daubechies. Orthonormal bases of compactly supported wavelets. Comm. Pure Appl. Math, 41:909-996, 1988.

[3] G. A. Elliott and D. E. Evans. The structure of the irrational rotation $\mathrm{C}^{\star}$-algebra. Ann. of Math, 138:477-501, 1993.

[4] O. Bratteli G. A. Elliott D. E. Evans and A. Kishimoto. Noncommutative spheres. ii. rational rotations. J. Operator Theory, 27:53-85, 1992.

[5] M. Frank and D. R. Larson. A module frame concept for Hilbert $C^{*}$-modules. Contemp. Math., $247,1999$.

[6] J. Cuntz G. A. Elliott F. M. Goodman and P. E. T. Jorgensen. On the classification of noncommutative tori ii. C. R. Math. Rep. Acad. Sci. Canada, 7:189-194, 1985.

[7] O. Bratteli G. A. Elliott F. M. Goodman and P. E. T. Jorgensen. Smooth Lie group actions on noncommutative tori. Nonlinearity, 2:271-286, 1989.

[8] Irving Kaplansky. Modules over operator algebras. American Journal of Mathematics, 75:839$858,1953$.

[9] E. Christopher Lance. Hilbert $C^{*}$-modules: A toolkit for operator algebraists. Cambridge Univ. Press, 1994.

[10] F. Luef. Gabor analysis, noncommutative tori, and Feichtinger's algebra. Lect. Notes Ser. Inst. Math. Sci. Natl. Univ. Singap., 10:77-106, 2007.

[11] S. G. Mallat. Multiresolution approximations and wavelet orthonormal bases of $L^{2}(\mathbb{R})$. Trans. Amer. Math. Soc., 315:69-87, 1989.

[12] J. Packer and M. A. Rieffel. Wavelet filter functions, the matrix completion problem, and projective modules over $C\left(\mathbb{T}^{n}\right)$. J. Fourier Anal. Applic., 9:101-116, 2003.

[13] J. Packer and M. A. Rieffel. Projective multi-resolution analyses for $L^{2}\left(\mathbb{R}^{2}\right)$. J. Fourier Anal. Applic., 10:439-464, 2004.

[14] William L. Paschke. Inner product modules over $B^{*}$-algebras. Trans. Amer. Math. Soc., 182:443-468, 1973 
[15] I. Raeburn and D. P. Williams. Morita equivalence and continuous-trace $C^{*}$-algebras. American Mathematical Society, 1998.

[16] M. A. Rieffel. Applications of strong Morita equivalence to transformation group $C^{*}$-algebras. Operator algebras and applications, Part I:299-310, 1980.

[17] M. A. Rieffel. $C^{*}$-algebras associated with irrational rotations. Pacific Journal of Mathematics, 93:415-429, 1981.

[18] M. A. Rieffel. The cancellation theorem for projective modules over irrational rotation $C^{*}$ algebras. Proc. London Math. Soc., 46:301-333, 1983. 